\title{
Gait transition and modulation in a quadruped robot: A brainstem-like modulation approach
}

\author{
Cristina P. Santos*, Vítor Matos \\ Industrial Electronics Department, School of Engineering, University of Minho, 4800-058 Guimaraes, Portugal
}

\section{A R T I C L E I N F O}

\section{Article history:}

Received 22 June 2010

Received in revised form

25 April 2011

Accepted 3 May 2011

Available online 13 May 2011

\section{Keywords:}

CPGs

Autonomous robots

Quadruped locomotion

Continuous gait switching

Architecture bio-inspired

Gait modulation

\begin{abstract}
A B S T R A C T
In this article, we propose a bio-inspired architecture for a quadruped robot that is able to initiate/stop locomotion; generate different gaits, and to easily select and switch between the different gaits according to the speed and/or the behavioral context. This improves the robot stability and smoothness while locomoting.

We apply nonlinear oscillators to model Central Pattern Generators (CPGs). These generate the rhythmic locomotor movements for a quadruped robot. The generated trajectories are modulated by a tonic signal, that encodes the required activity and/or modulation. This drive signal strength is mapped onto sets of CPG parameters. By increasing the drive signal, locomotion can be elicited and velocity increased while switching to the appropriate gaits. This drive signal can be specified according to sensory information or set a priori.

The system is implemented in a simulated and real AIBO robot. Results demonstrate the adequacy of the architecture to generate and modulate the required coordinated trajectories according to a velocity increase; and to smoothly and easily switch among the different motor behaviors.
\end{abstract}

(c) 2011 Elsevier B.V. All rights reserved.

\section{Introduction}

The ability to traverse a wide variety of terrains while locomoting is basically a requirement for performing useful tasks in our human centric world. However, legged locomotion is a complex problem that involves behavioral diversity. A common factor to these behaviors are the notions of trajectory generation and modulation. These are of utmost importance in robotics and animal control since trajectories have to be modified on-line in several circumstances, such as gait adaptation to speed change, obstacle avoidance, goal motion, or when dealing with external perturbations.

In this article we address some of the issues directly related to these two notions, considering the generation of different types of gaits and the easy switch between them in quadruped robots. Specifically, we address the topic of smooth gait transitions in a quadruped robot. We propose an open-loop controller architecture that must be able to generate different motor behaviors for a robotic quadruped, namely locomotion initiation, smooth gait generation and switching according to speed change and to stop locomotion. It takes its inspiration from biology [1-4] where a low dimensional tonic input from higher centers is used to control speed and direction of locomotion. This tonic command is used

\footnotetext{
* Corresponding author. Tel.: +351253 510190; fax: +351253 510189

E-mail address: cristina@dei.uminho.pt (C.P. Santos).
}

to modulate the parameters of CPGs in the lower level, modeled by non-linear oscillators, and thereby affecting gait in a smooth manner.

We believe that in order to devise flexible, adaptive, relevant locomotor models it is imperative to integrate concepts of the vertebrate locomotor generator structure organization, function, components and flexibility. However, our perspective is an engineering one and abstractions are done such that the proposed models are well suited for robots.

The present work extends ideas presented in [5-7] for the generation of other behaviors, namely in drumming and in switching between crawling and reaching. Herein, we further explore this idea for the generation and switching among other motor behaviors, continuing our previous work [8]. We propose an architecture structured in two functional hierarchical layers according to their level of abstraction.

The lower layer addresses the role of the spinal cord and generates the motor patterns by networks of Central Pattern Generators (CPGs). Based on previous work [8-10], we apply (oscillator-based) differential equations to model a network of four coupled unit-CPGs. These systems are solved using numerical integration and sent to the lower level PIDs of the joints.

The second layer models very basically the brainstem command centers for initiating, regulating and stopping CPG activity and therefore initiate locomotion, switch among gaits and stop the locomotion. This layer receives a modulatory signal and uses a piecewise continuous function to modulate the CPG parameters 
(frequency, amplitude and relative phases) according to the signal strength. By sending these at the right timing to the lower layer, it results in the modulation of the generated trajectories and thus in different motor behaviors.

Nature has provided solutions to the locomotion of animals which may, in some sense, represent an optimum, since it has evolved through millions of years. For instance, quadruped walking animals such as horses or cats change their gait to be suited to their walking speed. However, at all walking speeds the onset of swing in a foreleg occurs just after the onset of stance in the ipsilateral hind leg [1]. These facts are very important to realize smooth motor patterns.

In robotics, in order to achieve smooth walking from low speed to high speed, these gaits should be similarly switched continuously. This can be easily achieved by applying the wave gait rule [11,12], i.e., the interlimb phase relationships should follow the value of the duty factor for a changing speed. This rule improves the stability of the locomotion and maximizes the stability margin $[11,12]$ because the support of the body smoothly changes from three-point support (walk) to two-point support (trot) $[13,12,11]$.

In this article, we explore the idea of taking into account the speed change when switching from a walk to a trot gait, by continuously changing the duty factor and the interlimb phase relationships. These two factors fully characterize a gait and herein directly specify the CPG parameters of the locomotion controller. Because the resultant motor patterns are modulated according to modulation of the CPG parameters, this is easily and straightforwardly achieved using our formulation, and is one of our controller's main advantages.

The proposed system is implemented and tested in both, a simulated environment and the real ERS-7 robot from Sony. The obtained results demonstrate the adequacy and feasibility of the proposed locomotor controller to generate the required coordinated trajectories for locomotion; to modulate the generated motor patterns according to a velocity increase; and to smoothly and easily switch among the different motor behaviors. Further, both the modulation and switching are elicited according to a unique modulatory drive signal, either given a priori or by sensory information.

Results also show that the stability margin decreases approximately linearly with the velocity increase, and that the switching among the gaits happens smoothly. If instead an abrupt transition between these gaits is applied, the system moves quickly into a trot, exhibiting a non-natural, messy and less stable behavior.

This article is structured as follows. We will first review recent work on quadruped locomotion using Central Pattern Generators and gait transition. Section 3 presents details of motor patterns and gait transition. Section 4 briefly introduces the proposed architecture; discussing the neural structures involved in the locomotion of vertebrates; the biological observations more pertinent to this work and the controller requirements. Section 5 describes the lower layer of the proposed architecture. The second layer is presented in Section 6 where the mechanism to encode movement specifications is described in detail. Section 7 presents the obtained results for several experiments. We conclude by presenting the conclusions and discussing some future directions for our work.

\section{State-of-the-art}

In this work, we address the problem of developing a controller architecture, modeled by nonlinear dynamical systems, inspired in the functional model of biological motor systems that can online generate and modulate different motor patterns (gaits) and select and switch between them according to the (sensed) speed and/or the behavioral context for a quadruped robot.
We choose this task because it requires important features of movement control, notably timing, synchronization and accuracy and behavior integration. Experiments have been delineated such that these behaviors are integrated and their switch is elicited either by sensory information or a priori.

The design of the architecture takes into account experimental knowledge about how the nervous system deals with the control problem in a robust and flexible way [14,15,3,16-18]. It is partly inspired from the biological concepts of Central Pattern Generators (CPGs) [15], i.e., spinal-neural networks capable of autonomously producing coordinated rhythmic output signals; and by the concepts of force fields [19].

We have applied the dynamical systems theory for: (1) generating complex movements that smoothly superimpose and/or switch between discrete and rhythmic primitives; (2) easily control the switch between the possible movements; (3) DOFs coordination; and (4) modulate the movements according to given signals or possible feedback pathways.

The dynamical systems approach has proven to be successful in many robotic applications [20,14,21,5,22-24,9,25,8,7,26,27]. It offers multiple interesting features which apply well to model CPGs for robotic controllers, including: low computational cost; the intrinsic stability properties allow for feedback integration; intrinsic robustness against small perturbations; smooth trajectories modulated by simple parameter changes; provide for coupling/synchronization; and entrainment phenomena when coupled to mechanical systems.

Additionally, in the design of the architecture we have assumed that complex movements are constructed out of the combination of simpler motor primitives $[14,19,16]$. This modularity is also assumed in terms of behaviors [16]. In animals, different motor behaviors, designed to solve a variety of motor tasks, are stored as motor programs in the nervous system. Once activated, these are subject to parameterization that modulate the trajectories generated by the CPGs and produce different motor behaviors $[19,3]$.

These two last assumptions enable movements to be generated in a modular fashion and are convenient for modeling purposes [21]. This allows: (1) to tackle the complexity inherent to the design of dynamical systems; (2) a fast response to stimuli; and (3) an easy switching between behaviors. Thus, it is well suited for fast adaptive behaviors because it turns a high dimensional trajectory generation problem into a simple selection between predefined behaviors. This is in fact an interesting way of making the encoding of multiple trajectories more compact [28].

The proposed architecture is organized onto hierarchical layers, similarly to the motor control systems involved in goal-directed locomotion in vertebrates [3]. This modularity between the layers enables us to achieve independence between them which is adequate for a real implementation of the architecture, from a computational perspective. Higher layers that require more computational power but with larger time scales can easily be implemented in external computers and communicate with the robot when needed [28].

Control approaches based on CPGs and nonlinear dynamical systems are widely used in robotics to achieve tasks which involve rhythmic motions such as biped and quadruped autonomous adaptive locomotion over irregular terrain [29,22], juggling [30], drumming [8], playing with a slinky toy and basis field approaches for limb movements [19]. Some of these works present a high degree of sensor-driven and/or learned autonomy.

Herein, we extend the ideas presented in [7] for a similar architecture towards the achievement of continuous gait transition using a CPG-based approach. We do not expect precise and exact motions, since the CPG approach is not intended for such a goal and in this work is open-loop and disregards physical 
effects and other disturbances, which should be used to improve the locomotion's performance. In order to achieve (dynamical) stable locomotion, feedback control would have to be included. Despite the importance of such autonomy, we are particularly interested in completely understanding and exploring the trajectory generation problem in an open-loop manner. Additionally, other important considerations related to autonomous gait generation and switching such that the robot effectively learns to obtain different gaits by itself while interacting with its environment are outside the scope of the work herein presented.

Given the simplicity and the open nature of the architecture, we expect to verify the adequacy of the controller, similarly to its biological counterparts, to generate and modulate motor patterns without sensory feedback and without any rhythmic inputs, when activated by simple commands that somehow encode their rhythmic activation, frequency and amplitude. We therefore explore the open-loop features of the controller that are relevant to the control of a robotic platform as follows.

Stability and smoothness enable more natural movements, reduce the risk of the robot losing stability while locomoting and the risk of damage. Smooth movements diminish the strain in the robot joints.

Simple parameter changes in the controller should be able to generate different types of motor behaviors and the switch among these. Further, it must be possible to smoothly and predictably modulate the trajectories in order to meet the locomotor commands. In order to cope with adaptation to the environment it must be possible to modulate the generated movement according to the time-varying sensory information. Therefore, the controller must be stable and reliable. It must be stable to small perturbations, with the possibility of dealing with deviations in results and enabling feedback integration, making the locomotion more robust.

We have been progressively advancing, focusing on the open-loop aspects of the locomotion generation, such as the required motor primitives [8]; gait transition [10]; postural control [9], locomotion-induced head movement minimization [31], and currently we are addressing the inclusion of sensoryfeedback. Previously, the team developed some work in which sensory-motor information has been integrated in a dynamical architecture to generate timed trajectories [25,26]; coordinate robot behaviors [26,27,32]; and achieve steering and obstacle avoidance [25,27].

Several computational studies of gait transition exist but some of them have not been applied to robotics and others did not include the higher centers stimulation role in gait transition. Transition from the crawl to both the trot and pace gaits is described in $[33,34]$. However, these transitions are artificial and achieved in a feedforward manner. [35] describes a distributed approach to achieve smooth transitions from the crawl to the trot gait, and from the trot to the gallop gait. However, the approach is restricted to a small number of legs and broken legs may provoke non-smooth behavior, since legs influence each other through the interaction.

In [36] they incorporate distal sensor information into the gait selection problem and therefore address the coupling of the environment with the robot. In [37] they use CPGs integrated with higher commands, using ANN, to control sitting and lying down, and scratching. However, they abruptly switch between walking, trotting and galloping. [38] apply nonlinear oscillators to achieve a continuous gait transition. They do not, however, use rhythmic and discrete primitives. In [39] they use a neural network (Cerebellar Model Articulation Controller) to train 3 gait transitions. They do not use, however, CPGs. [40] present a gait coordination algorithm, responsible for inter-leg communication, that initiates leg state transitions and produces a stable gait. However, no detail is provided. In [41] they use a gait generator, and select between several gaits and omnidirectional gaits. However, they do not use CPGs neither do they care about a smooth transition.

Inagaki proposes and implements a continuous gait switching from the crawl to the trot gait [12]. Despite the excellent results, the robot mechanical structure is specifically designed to be suited for the control strategy. Freyr also describes the problem and develops the idea but only explores the statically stable crawl gait [13].

In [42] CPG-based gait transitions are explored in a salamander robot. The proposed model of neural mechanisms for modulation of velocity, direction and type of gait have inspired the current work. In [43] an interesting and similar network is presented to achieve a gait transition by varying a single parameter. However, in this work they used simple amplitude controlled phase oscillators. Besides, they have not considered that interlimb phase relationships should follow the value of the duty factor if a smooth transition between gaits is required and this gait transition is suitable to the locomoting speed.

The novelty of this contribution compared to other works is threefold: first it proposes a methodology to modulate the CPG's parameters that reduces the control dimensionality; second it generates, selects and switches between gaits and therefore increases behavioral diversity compared to previous CPG-based work [7,29,44]; and third it requires the tuning of a smaller number of parameters if compared to other CPG-based methodologies [29], having a straightforward outcome in the resulting trajectories. As canonical subsystems we have used nonlinear oscillators that are simple to treat analytically which makes it possible to build networks by coupling several of these subsystems and achieve the desired behavior.

The proposed controller is simpler when compared to other solutions [29], it enables us to elicit behavior switching, provides for a functional description, and improves the stability and the response of the robot during its locomotion, for the various velocities and can easily be used in different platforms.

\section{Gait characterization and transition}

Under some conditions of motion, a certain gait is more adequate for reasons that are related to stability, speed, energy efficiency, terrain properties, mobility or structure of the animal [1]. However, at all walking speeds the onset of swing in a foreleg occurs just after the onset of stance in the ipsilateral hind leg.

Similarly to its biological counterparts such as the cat, for example, we want that as the speed increases, the robot continuously changes from a crawl gait to a trot gait, in order to achieve a smoother quadruped locomotion. Thus, relative phases among the limbs should not be abruptly changed. Rather, phase relationships between diagonal limbs should gradually reach synchronization with an increase of speed (decrease of duty factor) [1].

Consider as the initial gait a creeping slow one: the crawl gait. This is a statically stable regular symmetric gait in which a leg in the air is set down (event $\varphi$ ) before the next one is lifted (event $\psi$ ), with at least three legs in ground contact at all times. Accordingly, the gait event sequence and its timing can be defined using the duty factor $\beta$, and the relative phase of the left hind leg, $\varphi_{\mathrm{LH}}$, called the gait phase from now on to simplify reading. The relative phases for all the limbs are given by

$$
\begin{aligned}
& \varphi_{\mathrm{LF}}=0, \\
& \varphi_{\mathrm{RF}}=0.5, \\
& \varphi_{\mathrm{RH}}=\varphi_{\mathrm{LH}}-0.5 .
\end{aligned}
$$




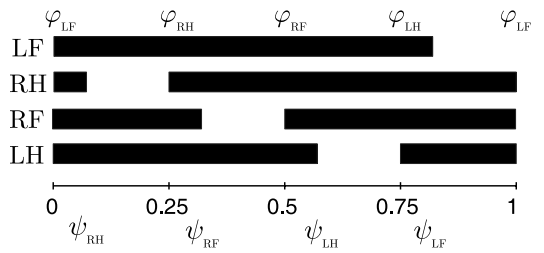

(a) Non-singular crawl.

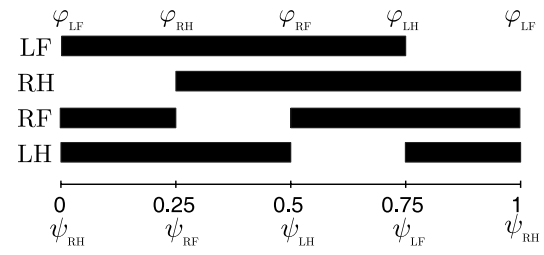

(b) Singular crawl.

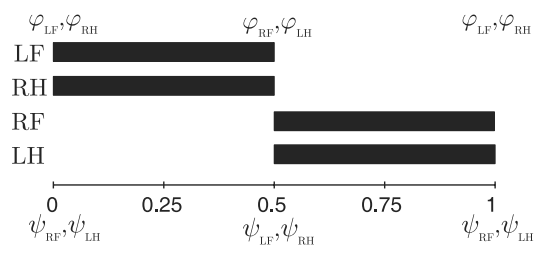

(c) Trot.

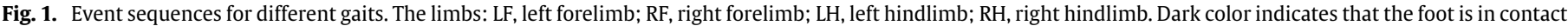
with the ground.

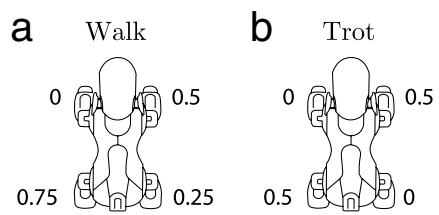

Fig. 2. Relative phases for the walk (a), and trot gaits (b).

In order to have three legs in ground contact at all times, it is further required that the duty factor fulfills $0.75 \leq \beta \leq 1$ and the gait phase $1.5-\beta \leq \varphi_{\mathrm{LH}} \leq \beta$. Fig. 1(a) shows the gait event sequence for this non-singular crawl gait, with the gait event sequence given by

$\left\{\varphi_{\mathrm{LF}}, \psi_{\mathrm{RH}}, \varphi_{\mathrm{RH}}, \psi_{\mathrm{RF}}, \varphi_{\mathrm{RF}}, \psi_{\mathrm{LH}}, \varphi_{\mathrm{LH}}, \psi_{\mathrm{LF}}\right\}$.

Relative phases among the limbs and gait diagram for this gait are depicted in Fig. 2(a) and Fig. 3(a), respectively.

Stability margin is maximized if a front leg is lifted at the same instant as the ipsilateral hind leg is set down [11]. In order to achieve this, we use the wave gait rule: the gait phase $\left(\varphi_{\mathrm{LH}}\right)$ follows the value of the duty factor $(\beta)$.

This results in a singular crawl gait, with relative phases given by

$\varphi_{\mathrm{LF}}=0, \quad \varphi_{\mathrm{RF}}=0.5, \quad \varphi_{\mathrm{LH}}=\beta, \quad \varphi_{\mathrm{RH}}=\beta-0.5$.

The corresponding gait diagram is depicted in Fig. 3(b). Basically, this maximizes the number of times there is ground contact of three legs, by placing and lifting subsequent limbs at the same time (both ipsilateral or contralateral).

The use of this rule is twofold. First, it provides for a crawl gait with maximized stability margin. Second, it provides a smoother transition from the crawl gait to trotting since it improves the stability of the locomotion due to the fact that the support of the body smoothly changes from three-point support (walk) to twopoint support (trot) $[13,11,12]$.

As the speed increases, the duty factor decreases and falls outside the creeping gait region. When the duty factor is decreased from 0.75 to 0.5 , the gait smoothly transfers from a singular crawl to a trot, the main difference being that the hind legs are lifted before their diagonal front legs in the air are set down. The gait event sequence and the corresponding gait diagram for $\beta \approx 0.6$ are depicted in Fig. 4. There are instants of time at which the robot relies upon two legs only.

Fig. 5 depicts the event sequences when no wave gait rule is applied. On the one hand, when the duty factor is 0.5 (necessary for a trot) but the gait phase is 0.75 (required for a walk), we can verify that the robot is at all times with only two feet in support (Fig. 5(a)). However, some phases of the stride correspond to a pace gait, in which two ipsilateral legs are in ground contact, instead of having the diagonal limbs in ground contact as expected in a trot. On the other hand, if $\beta=0.75$ but the gait phase is 0.5 (set according to a trot gait) (Fig. 5(b)), there are times in which four legs are in ground contact while at other times only two legs are in ground contact. Because this is a slow gait it is expected to be as much statically stable as possible, and as such it should have as far as possible three legs in ground contact. These comparisons explicitly show the advantages of applying the wave gait rule during speed change.

A trot gait is usually defined as a symmetric gait with duty factor ranging from $0.3-0.5$ and relative phases given by

$\varphi_{\mathrm{LF}}=0, \quad \varphi_{\mathrm{RF}}=0.5, \quad \varphi_{\mathrm{LH}}=0.5, \quad \varphi_{\mathrm{RH}}=0$.

Fig. 1(c) shows the trot gait event sequence, which is given by

$\left\{\varphi_{\mathrm{LF}} \varphi_{\mathrm{RH}}, \psi_{\mathrm{RF}} \psi_{\mathrm{LH}}, \varphi_{\mathrm{RF}} \varphi_{\mathrm{LH}}, \psi_{\mathrm{RH}} \psi_{\mathrm{LF}}\right\}$

The trot relative phases among the limbs are depicted in Fig. 2(b). The corresponding gait diagram is depicted in Fig. 6 . The trot gait appears as the limit of the gait event sequence for a duty factor $\beta=$ 0.5 , in which there is a switch between two diagonal supporting legs such that these move synchronously.

\section{Bio-inspired architecture}

This section describes some fundamental aspects of the proposed controller architecture.

\subsection{Neural structures for locomotion in vertebrates}

Intensive biological research has provided a description of the neural basis involved in locomotion generation [2], adaptation [45] and goal-directed locomotion [3].

Basically, networks of Central Pattern Generators (CPGs) located at the spinal level, capable of autonomously producing motor patterns, i.e., coordinated rhythmic output signals, in vertebrate animals [46]. These networks of CPGs control much of the timing, pattern, amplitude and rhythm of muscle activation [46]. They are able to generate these complex patterns without sensory feedback and without any rhythmic inputs, when activated by simple commands that encode their rhythmic activation, frequency and amplitude. This means that the repetitive patterns of activation for the limb muscles are generated without any peripheral input or rhythmic activation. Instead, CPGs are activated through tonic signals from supraspinal regions.

It has been proposed that the CPG for each limb is composed by smaller rhythmogenic circuits, the unit-CPG, each controlling one muscle antagonistic pair of a limb, i.e., one unit-CPG controlling one joint in a limb $[47,3]$.

Quadrupedal mammals locomote with several different gaits, requiring a certain level of coordination among the limbs. This interlimb coordination takes place at the CPG level, where the CPGs are tightly coupled among each other ensuring the precise activation of the muscles $[1,46]$.

Signals from supraspinal, spinal and peripheral structures are continuously integrated by the CPG networks for the proper expression and short-term adaptation of locomotion, providing a great versatility and flexibility in the performed movements $[48,45]$. In summary, CPGs provide the basic rhythm output 


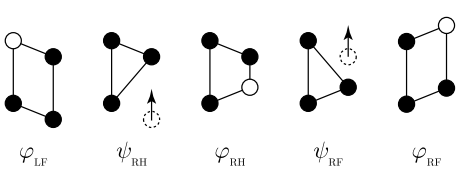

(a) Non-singular crawl gait.
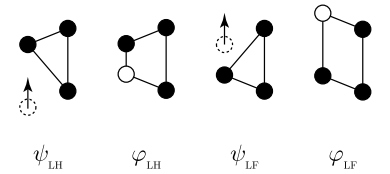

$\varphi_{\mathrm{LF}}$

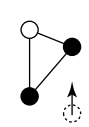

$\varphi_{\mathrm{LF}}=\psi_{\mathrm{RH}}$
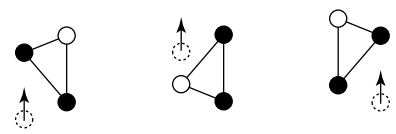

$\varphi_{\mathrm{RH}}=\psi_{\mathrm{RF}}$

$\varphi_{\mathrm{RF}}=\psi_{\mathrm{LH}}$

$\varphi_{\mathrm{LH}}=\psi_{\mathrm{LF}}$

$\varphi_{\mathrm{LF}}=\psi_{\mathrm{RH}}$

(b) Singular crawl gait in which $\varphi_{\mathrm{LH}}$ follows the value of the duty factor $\beta=0.75$.

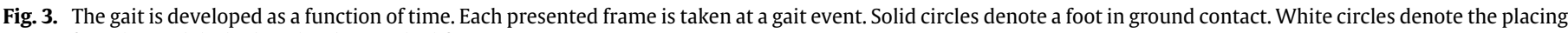
event of one leg and dashed circles denote the lifting event.

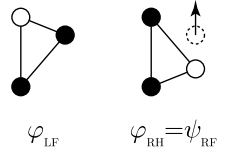

(a) Gait diagram.
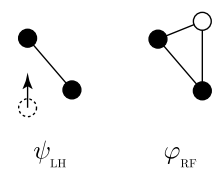

$\varphi_{\mathrm{RF}}$

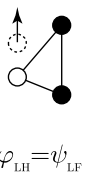

$\varphi_{\mathrm{LI}}=\psi_{\mathrm{LF}}$

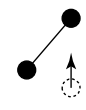

$\psi_{\mathrm{RII}}$

Fig. 4. Crawl gait with a duty factor $\beta \approx 0.6$, following the wave gait rule proposed by Inagaki and Kobayashi [12].

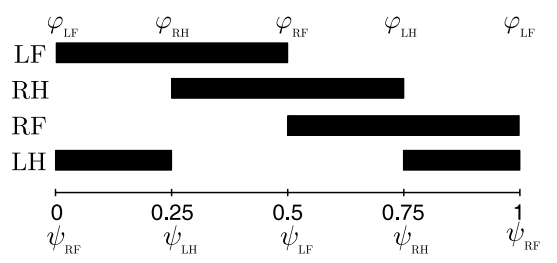

(a) $\beta=0.5, \varphi_{\mathrm{HL}}=0.75$.

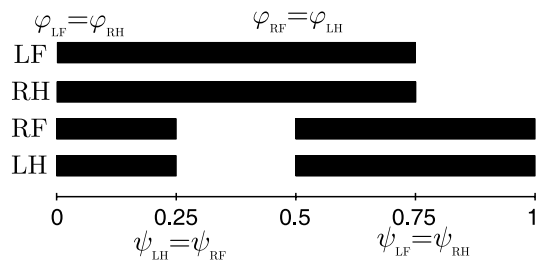

(b) $\beta=0.75, \varphi_{\mathrm{HL}}=0.5$.

Fig. 5. Example of gait diagrams when no gait rule is applied.

for locomotion while integrating powerful commands from various sources that serve to initiate or modulate its output, meeting the requirements of the environment.

The initiation and regulation of the activity of these networks is determined from the brainstem locomotor command systems, the mesencephalic locomotor region (MLR) and the diencephalic locomotor region (DLR). This regulation occurs via reticulospinal neurons (RS) [49]. Different stimulation strengths induce gait transitions, such that low stimulation gives rise to the slow walking gait and progressively greater strength will induce changes from walking to trotting to galloping. Meaning that, in quadrupeds, the speed of locomotion increases and there are changes in interlimb coordination.

This organization appears to be conserved throughout vertebrate evolution [3].

The nervous system contains several different motor behaviors, stored as motor programs, designed to solve a variety of motor tasks, from simple to complex. The basal ganglia is clearly implicated in selecting the appropriate motor program and to determine how different motor sequences are timed. Once a pattern of motor behavior is selected, the correspondent inhibition is relieved, and in the locomotion behavior case, the locomotor center in the brainstem is activated.

Once activated, these motor programs will produce different types of motor tasks.

\subsection{Relevant biological features}

Based on extensive research in the underlying locomotor systems of animals, it is possible to construe several required biological features that must be included in the locomotor controller in order to achieve locomotion and potentiate the desired flexibility and adaptability presented by animal locomotion. In summary, these are:
- Self-contained rhythmic generation per joint, the unit-CPGs, modulated by simple commands that encode their rhythmic activation, frequency and amplitude.

- Independent control of ascending and descending parts of the unit-CPG solutions, meaning an independent control of the swing and stance step cycle phase durations, for achieving different locomotor velocities.

- Coordination between the joints of a limb in order to correctly generate the locomotor movements and the desired gaits.

- Phase relation of the diagonal limbs gradually reach synchronization with the increase of speed.

- Pattern modulation and activation through simple commands from higher centers;

\subsection{Proposed architecture}

Similarly to the motor control systems involved in goaldirected locomotion in vertebrates [3], the proposed architecture is structured in functional hierarchical layers according to their level of abstraction. Herein, two layers are described and used. A parallel between a simplified biological architecture and the proposed one is presented in Fig. 7.

The lower layer addresses the role of the spinal cord and generates the motor patterns by networks of CPGs. It generates and coordinates the movements of the limbs in order to achieve the locomotor movements.

This layer receives sets of CPG parameters which generate different motor behaviors according to their timing. This set of parameters and their timing define a motor program. The addressed motor patterns are locomotion initiation, gait switching and to stop locomotion.

The second layer models very basically the brainstem command centers for initiating, regulating and stopping CPG activity and 


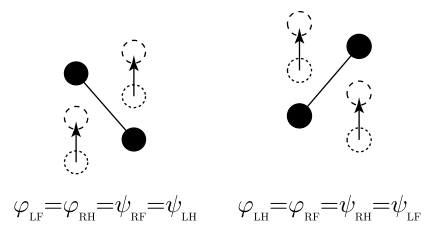

Fig. 6. Gait diagram for the trot gait.

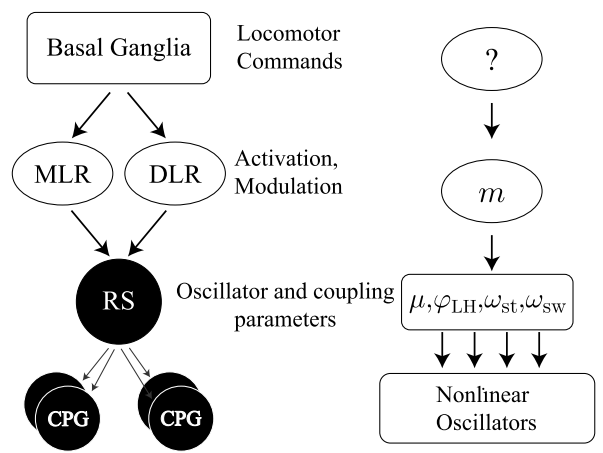

Fig. 7. Functional division of the motor controller structures in the nervous system of vertebrate (left), and the proposed locomotor controller architecture (right). The lower level generators receive excitatory and modulatory signals (parameters). Commands from higher centers and/or information from sensorial centers are mapped onto these parameters. Modules not addressed within this work have been labeled '?'.

therefore initiate a walking gait, switch among gaits and stop the locomotion. It selects different sets of CPG parameters and sends them, at the right timing, to the CPG network.

In the next sections, these two layers are described in greater detail.

\section{Lower layer: pattern generation}

The lower layer of the proposed architecture models networks of unit-CPGs that generate the motor patterns required for locomotion. The different generated motor patterns are initiated, modulated and switched by simple command signals which are the input of the layer.

We apply dynamical oscillators to model the CPGs. The dynamical systems approach $[29,50]$ applies well to model CPGs for robotic controllers due to their intrinsic properties discussed in Section 2. The dynamical systems have often proven to ensure a robust control of the movements in time-varying environments.

\subsection{Unit-CPG}

The rhythmic locomotor movements for a robot joint are generated by the $x$ variable of the following Hopf oscillator

$\dot{x}=\alpha\left(\mu-r^{2}\right) x-\omega z$,

$\dot{z}=\alpha\left(\mu-r^{2}\right) z+\omega x$,

where $r=\sqrt{x^{2}+z^{2}}$, amplitude of the oscillations is given by $A=\sqrt{\mu}$ for $\mu>0, \omega$ specifies the oscillation frequency (in rad. $s^{-1}$ ) and relaxation to the limit cycle is given by $\frac{1}{2 \alpha \mu}$.

This oscillator contains a Hopf bifurcation from a stable fixed point at $x=0$ (when $\mu<0$ ) to a structurally stable, harmonic limit cycle, for $\mu>0$.

The generated trajectories through this oscillator can be summarized as

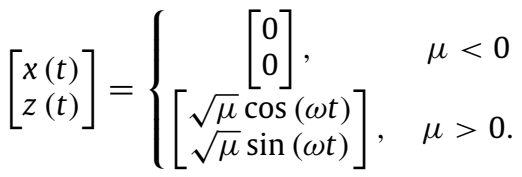

This oscillator generates smooth trajectories due to the stable solutions of the dynamical system, despite small changes in the parameters. We motivate the choice of this Hopf oscillator because it can be completely analytically solved, which facilitates the smooth modulation of the generated trajectories with respect to their amplitude and frequency (for speed change) according to small parameter changes, while keeping the general features of the original movements. Furthermore, it is able to modularly generate trajectories: discrete (i.e., no movement) and periodic.

Fig. 8 demonstrates the parameters' roles in the generated trajectories and in their modulation. The $\mu$ parameter generates discrete movement for $\mu<0$ and periodic movement for $\mu>0$ (Fig. 8(a)). Fig. 8(b) shows the fast but smooth amplitude modulation of the generated trajectories according to small changes in the $\mu$ parameter. Fig. 8 (c) depicts frequency modulations when the value of $\omega$ is slightly altered. The oscillator promptly changes the frequency and amplitude of the generated solutions, resulting in smooth and responsive trajectories.

This is interesting for trajectory generation in a robot [8] and for modeling the CPGs. The system is able to generate sustainable motor patterns without sensory feedback and without any rhythmic inputs, when activated by simple commands that somehow encode their rhythmic activation, frequency and amplitude. These features are similar to their biological counterparts.

The generated $x$ solution of this nonlinear oscillator is used as the control trajectory for a hip swing joint of the robot limbs. These trajectories encode the values of the joint's angles and are sent online for the lower level PID controllers of each hip swing joint.

Herein, we consider that the descending phase of the $x$ trajectory, in which the hip swing joint value is decreasing, corresponds to the stance step phase in which the limb moves backwards, thus propelling the robot forward. The ascending phase is the movement that places the foot in a more advanced position, ready for the next step, and corresponds to the swing step phase. This is depicted in Fig. 9(a). For $\omega>0$, when $z<0$ the limb is executing the swing step phase. When $z>0$ the limb is executing the stance step phase (Fig. 9(b)).

The oscillator described by Eq. (8) generates an $x$ oscillatory trajectory in which the ascending and descending parts have equal durations. In order to achieve an independent control of the duration of these parts, we employ the following equation proposed by [7],

$\omega=\frac{\omega_{\mathrm{st}}}{\mathrm{e}^{-a z_{i}}+1}+\frac{\omega_{\mathrm{sw}}}{\mathrm{e}^{a z_{i}}+1}$,

where $\omega$ alternates between two different values, $\omega_{\text {sw }}$ and $\omega_{\text {st }}$, depending on the step phase identified by the value of the $z$ variable. The alternation speed between these two values is controlled by $a$.

By controlling the durations of the ascending and descending phases of the $x$ trajectory, we are controlling the durations of the swing and stance step phases, respectively. This is achieved by setting $\omega_{\mathrm{sw}}=\frac{\pi}{T_{\mathrm{sw}}}$ (swing frequency) and $\omega_{\mathrm{st}}=\frac{\pi}{T_{\mathrm{st}}}$ (stance frequency).

It is thus possible to generate gaits with a desired duty factor, $\beta$, by keeping the swing frequency constant and specifying the stance frequency according to the duty factor value as follows,

$\omega_{\mathrm{st}}=\frac{1-\beta}{\beta} \omega_{\mathrm{sw}}$.

Fig. 10 demonstrates the generated solutions for two distinct duty factor values.

Inspired by neurobiology we name the unit-CPG the oscillator that generates movement for each hip swing joint. In summary, a 


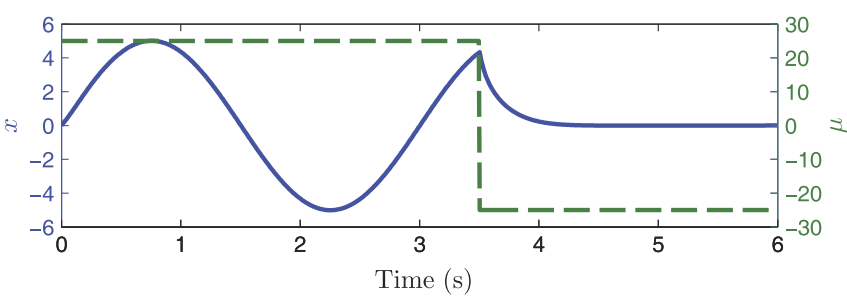

(a) The rhythmic solution is turned on for $\mu>0$ and turned off when $\mu<0$.

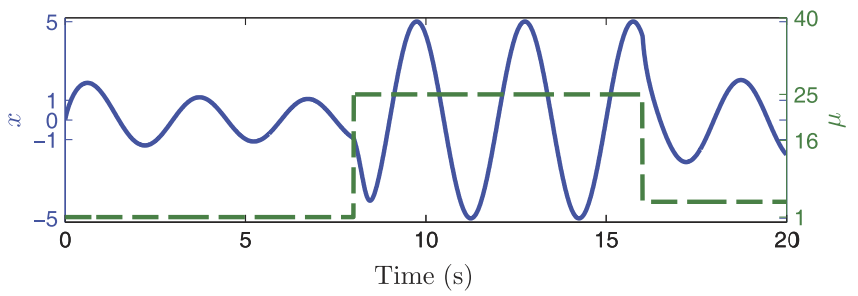

(b) The amplitude is modulated through the value of $\mu$. The solution's amplitude is given by $\sqrt{\mu}$.

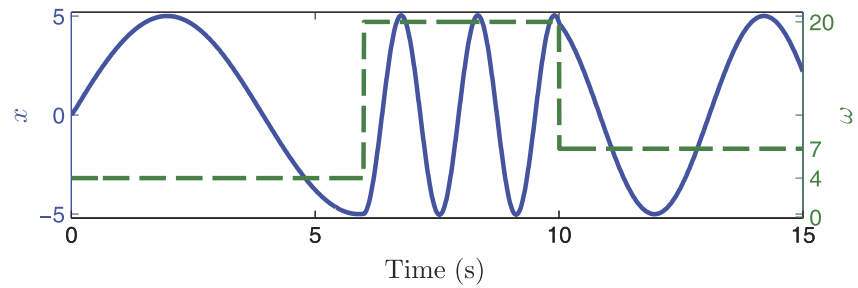

(c) Parameter $\omega$ controls the oscillation frequency for the rhythmic solution.

Fig. 8. Trajectory modulation and parameters' role. (a) Setting $\mu$ to a negative value turns off the oscillatory behavior due to the Hopf bifurcation, leading to no movement (discrete movement). Instead, if $\mu>0$ a purely rhythmic movement is obtained. (b) Amplitude modulation of the generated trajectories by small changes of the $\mu$ parameter (for $\mu>0$ ). (c) Small changes of the $\omega$ parameter modulates the generated trajectories in frequency.

a
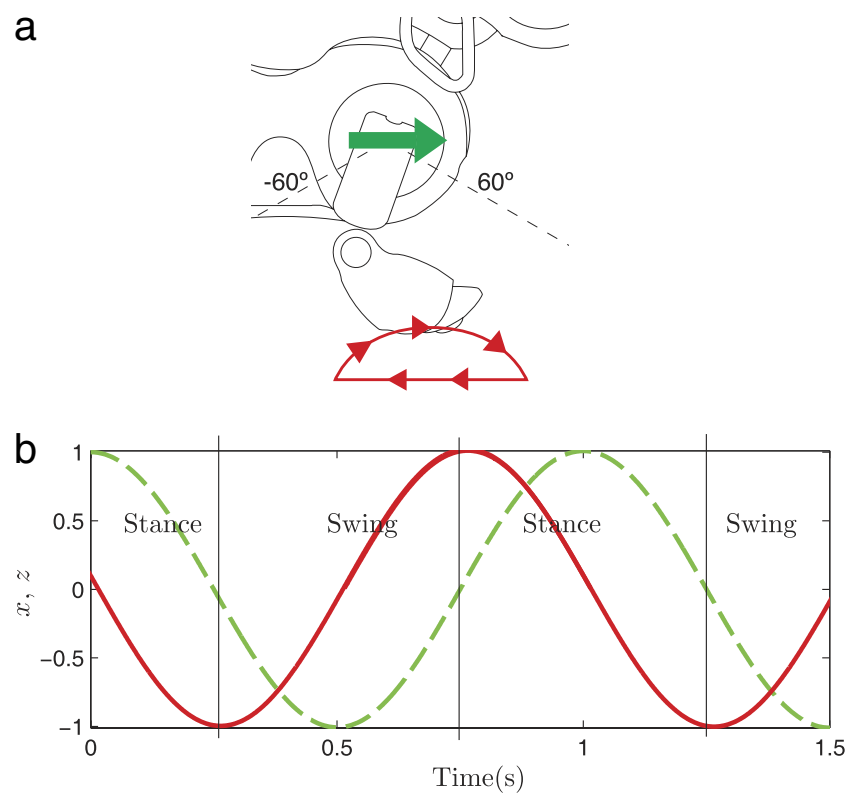

Fig. 9. (a) The robot is pushed forward when $\omega>0$. The corresponding movement of the descending trajectory, is the movement of pushing the robot forward. (b) $x$ trajectory (solid red line) is the control policy for the hip swing joint of the robot. The $z$ trajectory is represented by the dashed green line. Trajectories are obtained for $\omega>0$. When $z<0$, the robot is performing the swing phase movement, and the foot is placed in a more advanced position. When $z>0$, it is performing the stance phase. The limb moves forward, thus propelling the robot forward.

unit-CPG is given by

$\dot{x}=\alpha\left(\mu-r^{2}\right)(x)-\omega z$,

$\dot{z}=\alpha\left(\mu-r^{2}\right) z+\omega(x)$,

$\omega=\frac{\frac{1-\beta}{\beta} \omega_{\mathrm{sw}}}{\mathrm{e}^{-a z}+1}+\frac{\omega_{\mathrm{sw}}}{\mathrm{e}^{a z}+1}$,

with $r=\sqrt{x^{2}+z^{2}}$

Each unit-CPG takes a set of parameters for the modulation of its activity (Fig. 11), defining the joint's movement. These are

1. $\mu$, switches on/off the rhythmic output. If $\mu>0$ then it also encodes the amplitude of rhythmic activity, $\sqrt{\mu}$;
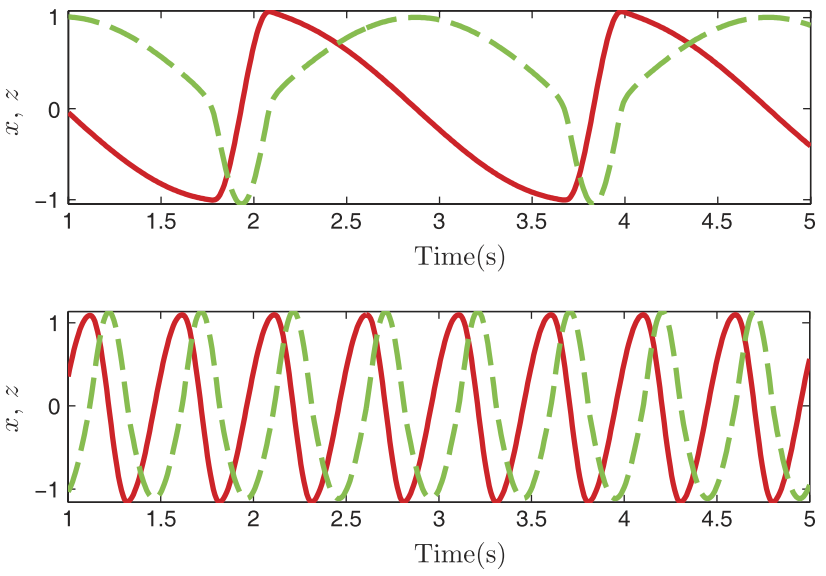

Fig. 10. Generated trajectories with $\beta=0.85$ (top) and $\beta=0.4$ (bottom): $x$ (solid red line) and $z$ (dashed green line). Trajectories obtained for a constant $\omega_{\mathrm{sw}}=10.4 \mathrm{rad} \mathrm{s}^{-1}\left(T_{\mathrm{st}}=0.3 \mathrm{~s}\right)$. Notice that the duration of the $x$ ascending phase (swing) is constant; only the duration of the $x$ descending phase (stance) changes.

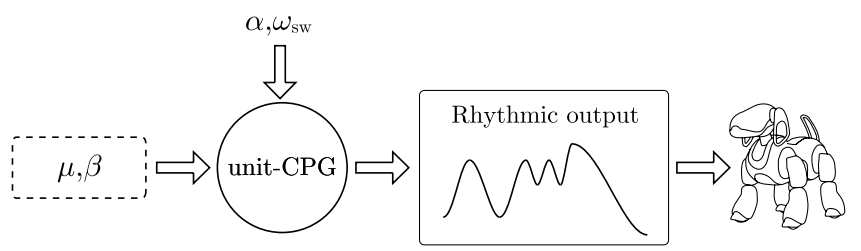

Fig. 11. Each unit-CPG receives a set of parameters that modulate the generated trajectories: (1) $\mu<0$, switch on-off the unit-CPG rhythmic activity, meaning that no periodic movement is generated; and (2) $\mu>0$ modulate the amplitude and frequency $\beta \in[0,1]$ of the unit-CPG rhythmic solutions.

\section{2. $\beta \in] 0,1[$, changes the walking velocity since it controls the stance duration of the generated movement.}

The parameters $\alpha, \omega_{\mathrm{sw}}$ and $a$ are set a priori. Parameter $\omega_{\mathrm{sw}}$ specifies the swing phase duration, which is kept constant. Its value depending on the desired speed of movement and on the robotic platform.

The knee joints are controlled as simply as possible: the knee is flexed to a fixed angle during the swing phase, and extended to a fixed angle during the stance phase. 


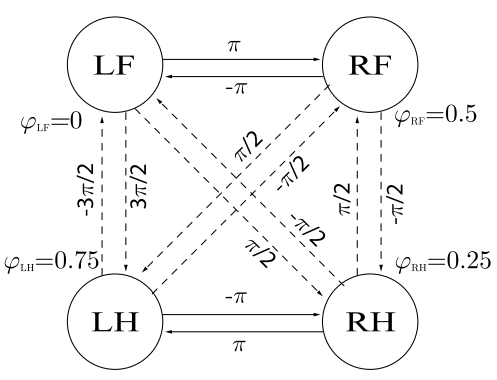

(a) Walk gait: each leg lags one quarter of a cycle in the sequence.

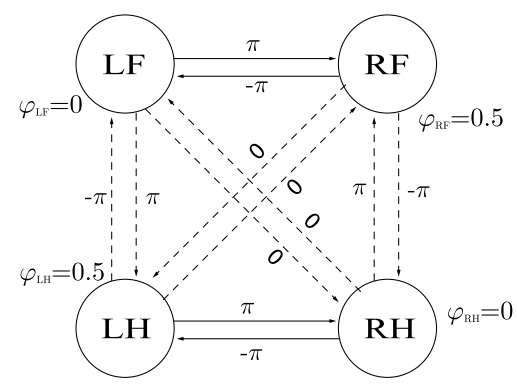

(b) Trot gait: diagonal legs move in synchrony while contralateral legs move in strict alternation.

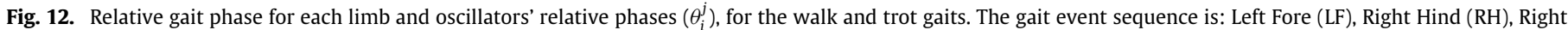
Fore (RF), Left Hind (LH). Oscillators are bilaterally coupled. Solid arrows represent the alternating relation, that does not change for symmetric gaits.

Table 1

Symmetric gait oscillators' phase relationships in terms of gait phase $\varphi_{\mathrm{LH}}$.

\begin{tabular}{lll}
\hline Generalized symmetric gaits & & \\
\hline$i$ & $j$ & $\theta_{i}^{j}$ \\
\hline LF & RF & $-\pi$ \\
RF & LF & $\pi$ \\
LF & LH & $-\varphi_{\mathrm{LH}} 2 \pi$ \\
LH & LF & $\varphi_{\mathrm{LH}} 2 \pi$ \\
LF & RH & $\left(0.5-\varphi_{\mathrm{LH}}\right) 2 \pi$ \\
RH & $\mathrm{LF}$ & $\left(\varphi_{\mathrm{LH}}-0.5\right) 2 \pi$ \\
RF & $\mathrm{LH}$ & $\left(0.5-\varphi_{\mathrm{LH}}\right) 2 \pi$ \\
LH & $\mathrm{RF}$ & $\left(\varphi_{\mathrm{LH}}-0.5\right) 2 \pi$ \\
RF & $\mathrm{RH}$ & $\left(1-\varphi_{\mathrm{LH}}\right) 2 \pi$ \\
RH & $\mathrm{RF}$ & $\left(\varphi_{\mathrm{LH}}-1\right) 2 \pi$ \\
$\mathrm{LH}$ & $\mathrm{RH}$ & $\pi$ \\
RH & $\mathrm{LH}$ & $-\pi$ \\
\hline
\end{tabular}

\subsection{Interlimb coordination}

In [1] it was discussed that at each gait speed it is possible to predict the angles in other joints from recordings of one joint. Hence, limbs behave as one unit and the movements in different joints are tightly coupled. Interlimb coordination is achieved by coupling in a given manner the dynamics of the four unit-CPGs, each controlling a hip swing joint. These couplings ensure that the limbs stay synchronized.

Unit-CPGs are coupled as follows

$$
\left[\begin{array}{c}
\dot{x}_{i} \\
\dot{z}_{i}
\end{array}\right]=\left[\begin{array}{cc}
\alpha\left(\mu_{i}-r_{i}^{2}\right) & -\omega \\
\omega & \alpha\left(\mu_{i}-r_{i}^{2}\right)
\end{array}\right]\left[\begin{array}{c}
x_{i} \\
z_{i}
\end{array}\right]+\sum_{j \neq i} \mathbf{R}\left(\theta_{i}^{j}\right)\left[\begin{array}{c}
x_{j} \\
z_{j}
\end{array}\right],
$$

where $i, j \in\{\mathrm{LF}, \mathrm{RF}, \mathrm{LH}, \mathrm{RH}\}$. The linear terms are rotated onto each other by the rotation matrix $\mathbf{R}\left(\theta_{i}^{j}\right)$, where $\theta_{i}^{j}$ is the required relative phase between the $i$ and $j$ hip swing oscillators to perform the gait (we exploit the fact that $\mathbf{R}(\theta)=\mathbf{R}^{-1}(-\theta)$ ).

The relative phases are specified according to the walk (Fig. 12(a)) and the trot gaits (Fig. 12(b)), in which contralateral legs always move in strict alternation.

These relative phase relationships between the oscillators can also be calculated according to the gaits' relative phases by

$\theta_{i}^{j}=\left(\varphi_{i}-\varphi_{j}\right) 2 \pi$.

The addressed gaits in this work are symmetric and as such always have $\varphi_{\mathrm{LF}}=0, \varphi_{\mathrm{RF}}=0.5$, and $\varphi_{\mathrm{RH}}=\varphi_{\mathrm{LH}}-0.5 \mathrm{Eq}$. (3). By substituting these values into Eq. (16), it is possible to express the oscillators' relative phases in terms of the gait phase $\varphi_{\mathrm{LH}}$, as presented in Table 1.

Interlimb coordination can then be achieved by specifying only the gait phase $\varphi_{\mathrm{LH}}$. During the transition from walk to trot, the diagonal limbs start to synchronize and the ipsilateral limbs move

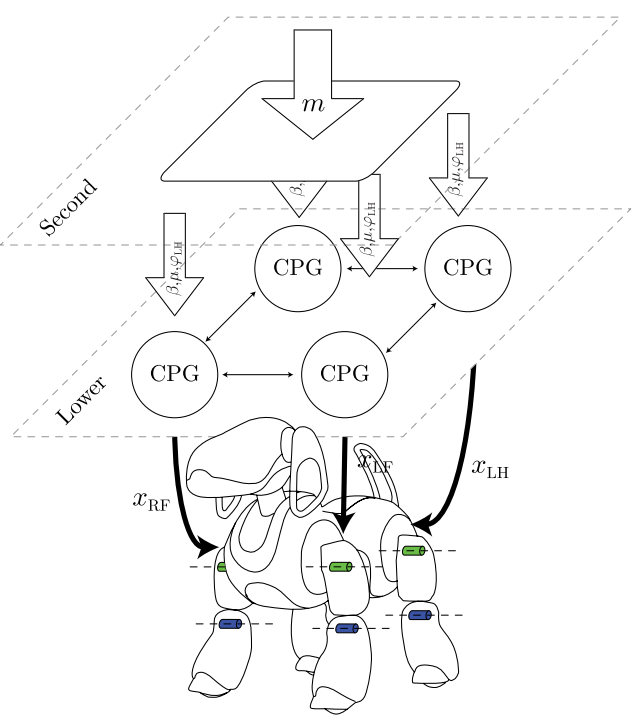

Fig. 13. Representation of the two levels of the architecture with the respective inputs and outputs. The second layer receives the activation signal $m$, relaying the information to the lower layer through the global and individual parameters of the CPGs. Each CPG receives the value for its phase relationship among the other CPGs through $\varphi_{L H}$ and its oscillatory activity through $\mu$. The stepping frequency of all the limbs is specified by the duty factor value of $\beta$. This lower layer outputs the joint angle information for the robot's hip PID controllers.

towards strict alternation. In order to achieve this, $\varphi_{\mathrm{LH}}$ is set equal to the duty factor value, $\beta$.

The final result is a network of oscillators with controlled phase relationships, able to generate more complex, synchronized behavior such as locomotion. Due to the properties of this type of coupling among oscillators, the generated trajectories are stable and smooth and thus potentially useful for trajectory generation in a robot.

This network of coupled unit-CPGs constitutes layer one of the proposed architecture (Fig. 13). It generates coordinated rhythmic movements in a stable and flexible way. The generated trajectories are smooth, stable and robust to perturbations.

It receives from the upper levels the required CPG parameters that modulate in a simple and straightforward manner the generated trajectories. These are $\mu, \beta$ and $\varphi_{\mathrm{LH}}$.

\section{Second layer: brainstem activation of motor programs}

We are interested in exploring the mechanisms that underlie gait transitions induced by simple electrical stimulation of the brain stem. The lower layer pattern generators are modulated according to sets of parameters that control their activity and 
modulation. These ideas were applied by [6] in the achievement of different behaviors, and are built upon the biological concept of a motor program [51]. The idea that sets of muscle commands are stored and launched with the right timing explains reactive responses to stimuli, for instance.

Different sets of CPG parameters correspond to different motor behaviors. However, bio-inspiration suggests that single tonic signals, from supraspinal regions, should somehow encode the required activity and/or modulation; providing a mapping from the tonic signals to the set of CPG parameters. Such mapping reduces the dimensionality of the control problem to just one excitatory signal. For instance, increasing activation of the brainstem locomotor center commands leads to an increase in quadruped locomotion speed and to a gradual shift in interlimb coordination, from walk to trot.

In our model, a given modulatory drive signal, $m$, models the brainstem locomotor center commands and regulates the activity of the unit-CPG network. Different strengths of this drive correspond to different motor behaviors, namely: locomotion initiation, stopping and increasing locomotion speed with adjustment of interlimb coordination.

Below a lower threshold, $m_{\text {low }}$, the robot ceases stepping. Above this threshold, the robot starts with a slow walk (non-singular crawl), gradually increasing speed without adjusting the phase relationships. Above $m=1$, locomotion speed is increased with adjustment of interlimb coordination. At $m=2.5$, the robot is in a trot. Herein, both the range and the thresholds for the modulatory drive were chosen arbitrarily.

This second layer is responsible for determining and sending the CPG parameters for the lower layer at regular time intervals such that the desired task is achieved. The task to be achieved, expressed in the value of $m$, may be defined either by an higher level or by sensory information.

\subsection{Initiating/stopping locomotion}

Qualitatively, by modifying on the fly the $\mu$ parameter, the system Eq. (8) switches between a stable fixed point at $x=0$ (for $\mu<0$ ) and a purely rhythmic movement (for $\mu>0$ ). Hence, the $\mu$ parameter controls whether or not there are oscillations generated by the unit-CPG and thus, locomotion generation. We consider that for $\mu<0$ the generated movement (that is relaxation to the fixed point) is in fact a discrete movement. The fixed point could be an offset that changes if it becomes the state variable of another dynamical system [8].

Below a lower threshold, $m_{\text {low }}=0.2$, the oscillators are shut down and the robot rests. However, for $\mu>0$ this parameter also encodes the amplitude of rhythmic activity. Above this threshold, $m \geq m_{\text {low }}$, there exists locomotor activity with amplitude $A$. $A$ is set $a$ priori in order to respect the robot feasibility.

The parameter $\mu$ is set as a piecewise linear function of the drive (Fig. 14(a)), such that

$\mu= \begin{cases}-A^{2}, & m<m_{\text {low }} \\ A^{2}, & m \geq m_{\text {low }} .\end{cases}$

\subsection{Robot velocity modulation}

The robot velocity is changed by changing the duty factor $\beta$, which results in controlling the stance phase duration: a smaller stance phase duration results in a higher velocity.

As the modulatory drive increases in strength, the duty factor $\beta$, linearly decreases from 0.89 (for the crawl gait) to $\beta=0.5$ (for the trot gait). The duty factor is mathematically defined as a piecewise linear function of the modulatory drive (Fig. 14(b))

$$
\beta= \begin{cases}0.89, & m<m_{\text {low }} \\ -0.1667 m+0.9167, & m_{\text {low }} \leq m<2.5 \\ 0.5, & m \geq 2.5\end{cases}
$$

This function presents a saturation for $\beta=0.5$ because the robotic platform can not perform faster gaits.

\subsection{Interlimb coordination modulation}

In this work, we want that in between $m_{\text {low }} \leq m<1$, the robot gradually increases its speed from a slow walk but without adjusting the phase relationships. For $\beta$ ranging between 0.89 and 0.76 , the robot presents a non-singular crawl gait with a constant gait phase $\varphi_{\mathrm{LH}}=0.75$.

For $1 \leq m \leq 2.5$, the crawl gait slowly transfers into a trot gait but adjusting the phase relationship accordingly. For that, we apply the wave gait rule for a quadruped: $\varphi_{\mathrm{LH}}=\beta$. In the resulting gait, subsequent legs are lifted closely after the previous ones are set down, such that the time difference between the two events is equal to zero. Hence, $m$ modulates the gait phases by specifying the gait phase $\varphi_{\mathrm{LH}}=\beta$.

The gait phase remains in $\varphi_{\mathrm{LH}}=0.5$ for values of the modulatory drive greater than 2.5 , which correspond to a $\beta$ of 0.5 (Fig. 14(c)). The robot is then with a trot gait.

\subsection{Behavior switching}

Different values of the drive lead to different behaviors, namely: locomotion initiation, speed change and consequent gait change, similarly to the biological counterparts [4]. Different behaviors correspond to different specifications of the set of CPG parameters: amplitude, duty factor and gait phase.

Herein, the switch among these possible behaviors is defined a priori but could be defined according to time-varying sensory information, e.g., external stimuli [6]. For instance, in the experiments section, we present an experiment in which a touch on the back of the robot initiates the walking pattern. A continuous touch on its back elicits speed change and gradual transition and a touch in the head elicits stopping the locomotion.

\section{Results}

To demonstrate the implementation of our model on a quadruped robotic system, we make several experiments both in simulation and in the real robot AIBO.

The AIBO dog robot is a 18 DOFs quadruped robot made by Sony. Unlike its natural counterpart this robot has three joints per limb, with different configurations of a real dog's limbs. Besides, the robot body and limbs are rigid with non-compliant servo joints. The joints are stiff, without any elasticity, and their position is specified by an angle value. The durations of the achieved step phases have to be limited, because the joints present timing constraints on movement executions.

Fig. 13 shows a view of the AIBO and the controlled DOFs. The CPG controlled joints are the hip swing joints (light green colour in Fig. 13) of each limb. Knee joints (dark colour in Fig. 13) are controlled according the hip CPGs, set at the right time to the correct values. The other DOFs remain fixed to an appropriately chosen value, such that the AIBO dog achieves a locomotion as reliable and stable as possible.

We set the frequency to $\omega_{\mathrm{sw}}=6.28 \mathrm{rad} . \mathrm{s}^{-1}$ with regards to the motor limitations. Further, the dynamical parameters controlling the speed of convergence of unit-CPGs were set to $\frac{1}{2 \alpha_{\mathrm{i}} \mu_{\mathrm{i}}}=0.09 \mathrm{~s}$, 


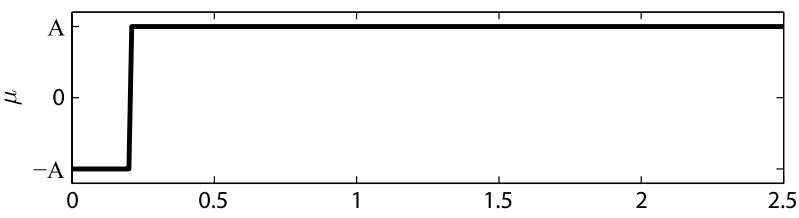

$m$

(a) Oscillator's parameter $\mu$. For a modulatory drive greater than 0.2 the oscillators are activated with amplitude $A$.

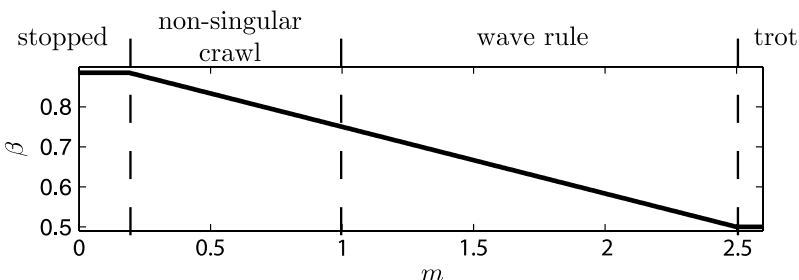

(b) Parameter $\beta$. The duty factor $(\beta)$ decreases linearly with the increase of the modulatory signal, until reaching 0.5 for an $m=2.5$.

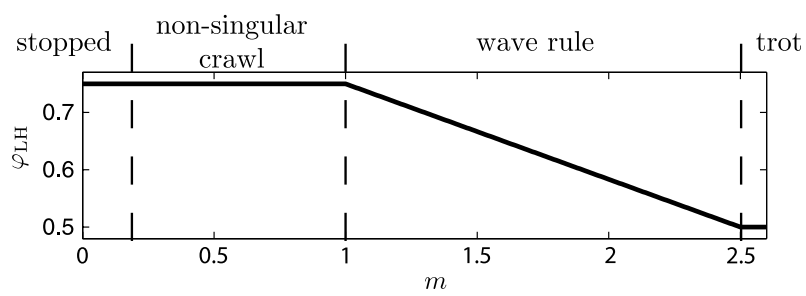

(c) Parameter $\varphi_{\mathrm{LH}}$. There is an adjustment of the gait phase according to the wave gait rule only for $1 \leq m \leq 2.5$.

Fig. 14. Effect of the modulatory drive $m$ on the unit-CPG parameters.

with regard to stability during the integration process and to feasibility of the desired trajectories.

At each sensorial cycle, dynamic equations are calculated and numerically integrated using the Euler method with a fixed time step of $1 \mathrm{~ms}$, thus specifying servo positions. The robot control loop is measured and is $32 \mathrm{~ms}$.

Webots [52] is a simulation software based on ODE, an open source physics engine for simulating 3D rigid body dynamics. One feature of Webots is to allow a user to control both a real Sony AIBO robot and its physical simulation. The same $\mathrm{C} / \mathrm{C}++$ code developed for the simulation is used to control the AIBO robot.

\subsection{Gradual vs abrupt gait change}

In this work we claim that gait transition from a statically stable, slow crawl gait to a faster trot gait should happen in a stable and smooth manner.

In this section, we will verify the robot behavior when going from a non-singular crawl gait to a trot gait, when the wave gait rule is and is not applied. Results will be evaluated and compared in terms of the robot velocity and stability margin. Also, changes in these terms will be compared based on the smoothness of their variation.

Firstly, it is necessary to further define what we consider by an abrupt gait change. An abrupt gait change from walk to trot occurs when, for $0.75>\beta>0.5$, the gait phase is abruptly adjusted from a walk (0.75) to a trot gait (0.5), as well as an abrupt change of the duty factor to 0.5 . This raises the question of when exactly should this change occur. That is, for which value of the duty factor should the gait phase be changed? In order to answer this question we delineated a series of simulations. The robot is initially walking with a singular crawl gait, with $\beta=0.75$ and $\varphi_{\mathrm{LH}}=0.75$. Duty factor is linearly decreased by 0.005 at each control loop cycle (32 $\mathrm{ms}$ ). The gait phase is abruptly changed from 0.75 to 0.5 at different values of $\beta$ in each simulation, namely $\{0.7,0.65,0.6,0.55,0.5\}$. When the gait phase is changed the duty factor is also set to 0.5 .

For measuring stability we calculate the wide stability margin [53] (WSM), that provides the shortest distance from the vertical projection of the center of gravity onto a horizontal plane, to an edge of the convex polygon formed by the vertical projection of the feet contact points onto the same horizontal plane. We measure and register the WSM and the velocity for each control loop cycle. This data is shown in Fig. 15.

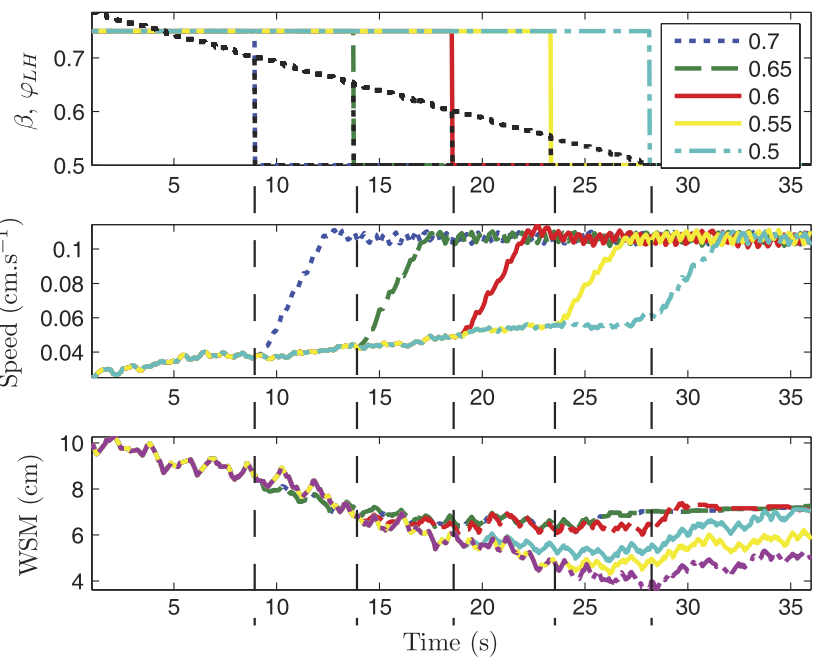

Fig. 15. Comparison results in terms of velocity and WSM, when abruptly changing from a non-singular crawl gait to a trot gait. $\beta$ varies from 0.75 to 0.5 . When $\beta$ reaches $\{0.7,0.65,0.6,0.55,0.5\}$ both the duty factor and the gait phase are abruptly changed, to 0.5 and from 0.75 to 0.5 , respectively. Different lines are used to indicate the different values of the duty factor when the abrupt change occurs.

Because these experiments are performed in simulation we know the robot position at each instant of time, and thus we are able to calculate the velocity. However, the velocity and WSM vary for each instant due to the stepping movement, and therefore we have filtered it using a simple running average filter.

The idea is that such an abrupt change should both smooth the increase in the velocity and maximize the WSM (that is, the amount of time the robot has larger WSM values). For faster speeds ( $\beta<$ 0.75 ), the walk of the robot is no longer statically stable and the gait phase of the walk is no longer adequate for the faster stepping frequency. The middle panel of Fig. 15 shows the robot speed. An abrupt change moves the system quickly into a trot gait, but with some instability in the robot behavior, which becomes somehow messy. This is difficult to demonstrate in our robot, because its physical construction does not enable us to exhibit gaits which are dynamically stable and would require some elasticity both in their limbs and body. In fact, the robot should mainly have contact of two feet, but the robot tends to fall over three legs instead due to its lack of elasticity. It is an unnatural movement, however. This 

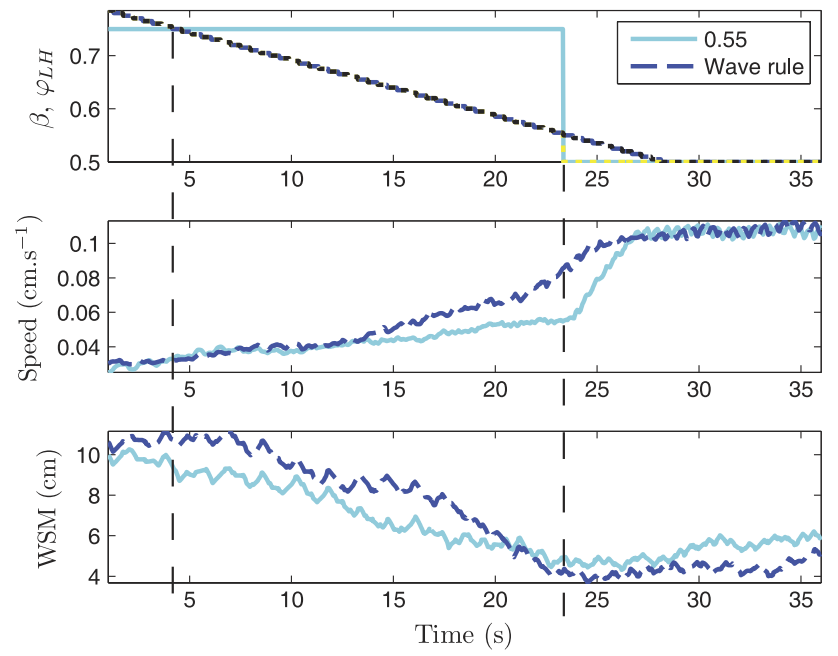

Fig. 16. Comparisons in terms of velocity and WSM when the wave gait rule is (dashed dark blue line) and is not (solid light blue line) applied. Top: duty factor $\beta$ and gait phase $\varphi_{\mathrm{LH}}$. Middle: locomotion velocity. Bottom: WSM. Locomotion velocity increases in a more smooth and controllable manner when the wave gait rule is applied. Also the WSM presents higher values for slower gaits when the rule is applied.

explains the WSM values (bottom panel of Fig. 15) after the abrupt phase gait changes. It exhibits large values in the trot gait because the robot falls over three legs.

In summary, an abrupt gait change from a crawl to a trot gait changes the system quickly into a trot gait but leading to some instability in the robot behavior (which is for some time quite messy), while the wave gait rule is expected to make this achievement to be accomplished in a more stable and smooth manner. Based on this data, we choose $\beta=0.55$ as the duty factor value at which the gait phase should be abruptly changed. This represents a compromise between the achieved WSM value and the velocity.

Two simulations are now performed: one using a continuous transition between gaits, and another using an abrupt transition between gaits. In both simulations, the robot walks forward during the first $4 \mathrm{~s}$ with a non-singular crawl gait (duty factor $\beta \geq 0.75$ and a gait phase $\varphi_{\mathrm{LH}}=0.75$ ). After $4 \mathrm{~s}$, it starts to slowly increase its stepping frequency by linearly reducing its duty factor by 0.005 each control loop cycle. Data from these simulations is shown in Fig. 16. Dark dashed and light solid lines are used to show data when the wave rule is and is not applied, respectively. We calculate the stability margin (bottom panel) and measure the robot walking velocity (middle panel). The top panel shows the duty factor and gait phase.

In the first simulation, after $4 \mathrm{~s}$, the robot continuously adjusts the gait phase according to the duty factor (top panel of Fig. 16, dotted light blue line). It reaches the trot gait only when both the duty factor and gait phase are 0.5 , at $t=28 \mathrm{~s}$.

In the second simulation, after $4 \mathrm{~s}$, the robot starts to decrease the duty factor and abruptly changes the gait phase from 0.75 to 0.5 when a duty factor of 0.55 is reached (top panel of Fig. 16, solid light blue line), at $t=23 \mathrm{~s}$.

Velocity (middle panel) changes in a more smooth way when gait phase varies according to the duty factor (dashed darker blue line). Note that velocity increases more when the rule is applied. At the moment of abrupt change, the velocity rapidly jumps to a higher value (solid lighter blue line). However, we have already discussed that the resultant behavior is messy and unnatural. This is relevant in a real robotics implementation, since the servos vary more gradually and are subjected to less strain.

As expected, there is an improvement in the stability measure for the slower gaits, where it matters the most, when there is a continuous switching (dashed darker blue line in the bottom panel of Fig. 16). The WSM presents higher values during the duty factor decrease when the rule is applied, meaning it is maximized. This happens since the wave gait rule maximizes the time that there is ground contact of three legs simultaneously. The transition is therefore accomplished in a more stable manner. Further, the stability margin decreases smoothly, i.e., gradually, as the gait is forced to a trot. In a real implementation, this smoothness may minimize the need for strong reactions in order to recover equilibrium and/or stable locomotion.

\subsection{Locomotor behavior switching}

In this experiment, we are interested in showing the easy switching between the different motor behaviors according to a modulatory drive, $m$, set a priori. Note, however, that fast online modulation of the trajectories is also verified during these switchings.

Fig. 17 shows an experiment in which the three motor behaviors are generated and modulated according to a gradual increase in the $m$ modulatory drive. Top panel depicts the $m$ drive.

Initially, the drive is below $m_{\text {low }}$ and the robot rests.

Around $t=10.5 \mathrm{~s}$, the linearly increasing drive crosses the $m_{\text {low }}$ threshold and locomotion is initiated with a non-singular crawl gait $\left(\beta \approx 0.88\right.$ and $\varphi_{\mathrm{LH}}=0.75$ ). The second panel of Fig. 17 shows the duty factor (light solid line) and gait phase (dark dashed line).

The drive keeps linearly increasing but is below 1 for the next $15 \mathrm{~s}$. Hence, the robot continues in the crawl gait, changing the duty factor but without gait phase adjustments. After $t=25 \mathrm{~s}$, the robot keeps increasing its velocity (because $m$ keeps increasing) but now gradually adjusting both the duty factor and the gait phase. At $t=$ $40 \mathrm{~s}$, the drive is abruptly set to its maximum (2.5). The gait evolves to a trot gait, with the ipsilateral limbs in strict alternation $\left(\varphi_{\mathrm{LH}}=\right.$ 0.5 ) and a duty factor of 0.5 . Servo ipsilateral left hip joints (black and light lines for fore and hind joints respectively) are shown in the fourth panel. Note the frequency and phase modulation. Because $\beta$ is decreasing, the stance frequency increases until reaching the trot. This is even more notorious when $m$ is abruptly set to 2.5. Further, the oscillators that initially lag a quarter of a cycle progressively are phase shifted until reaching anti-phase. Both these modulations are fast but smooth, as expected.

At $t=50 \mathrm{~s}$, the modulatory drive is set to zero, meaning that $\mu$ is set to a negative value (third panel) and the oscillators stop. Consequently, the locomotion is stopped. The bottom panel Fig. 17 shows the locomotion speed evolution.

Fig. 18 shows the gait diagram of the simulation, where the robot gradually transits from a slow walk to a faster walk, close to a trot. Note how subsequent limbs are lifted and set down almost simultaneously during the singular crawl. As the generated gait approaches a trot, since the controller doesn't correct or adapt for the dynamical gait, the robot does not perform perfectly.

Some snapshots of the three motor patterns for times ranging from 14 to $16 \mathrm{~s}$ (the non-singular crawl), 27 to $29 \mathrm{~s}$ (singular crawl), and 44 to $45 \mathrm{~s}$ (trot gait) are depicted in Fig. 19. The two first rows of snapshots correspond to the instants marked by gray lines in Fig. 18. The corresponding video can be seen in [54].

\subsection{Real robot}

In this section, we want to verify the suitability of the architecture for the generation and modulation of different motor behaviors. Each of the different motor behaviors, i.e., the nonsingular crawl, singular crawl and trot gaits, require the use of the four limbs and the correct coordination between the limbs. Further, the online modulation of the generated trajectories is required 


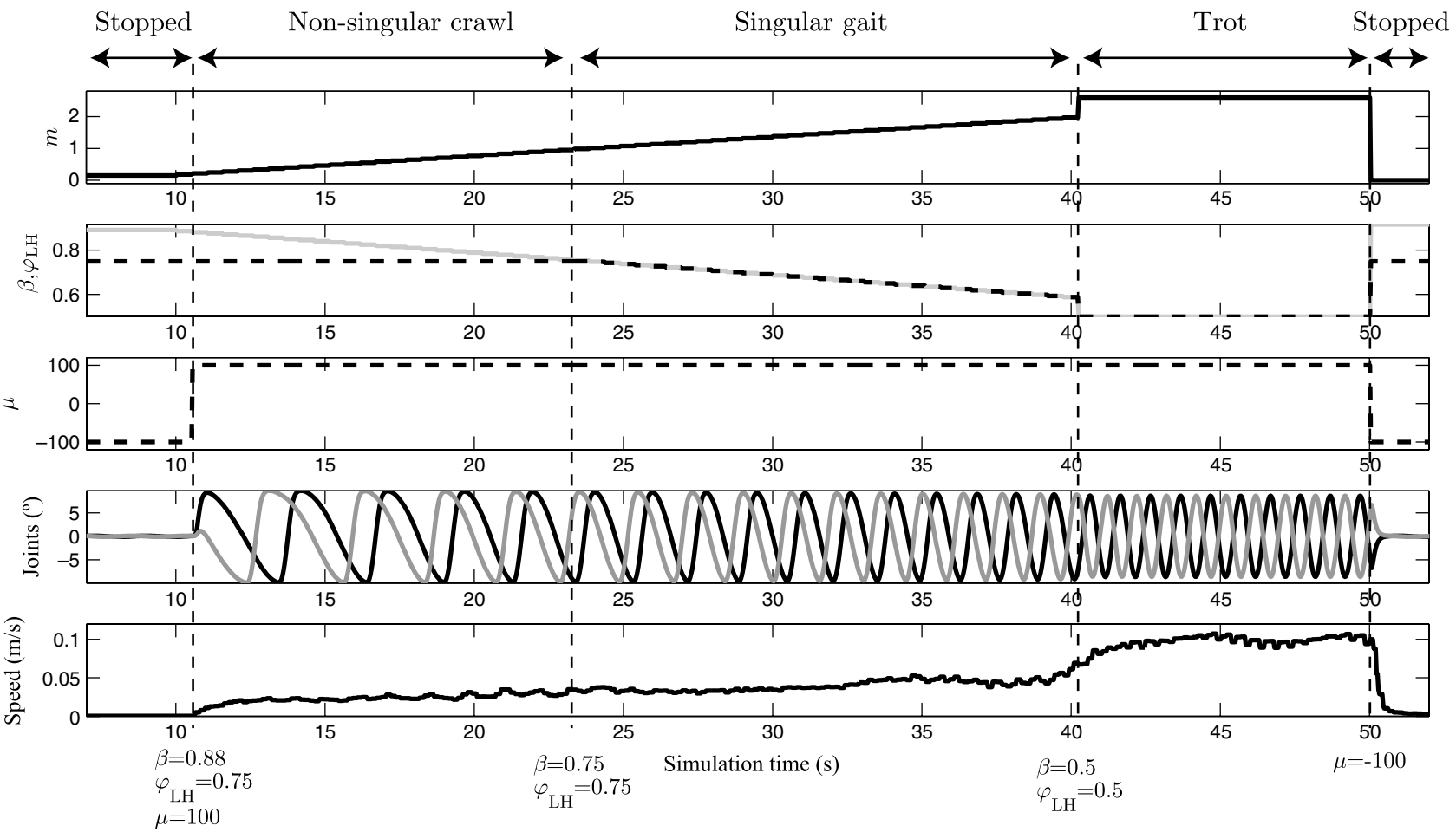

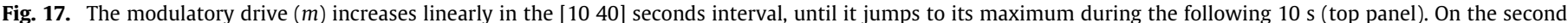

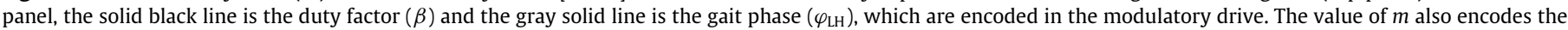

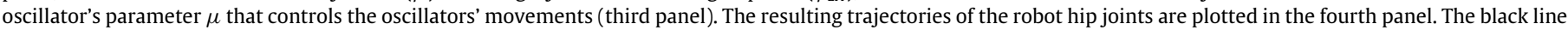

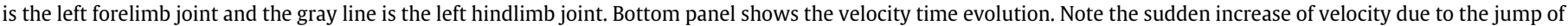
the modulatory drive at $t=40 \mathrm{~s}$. Around $t=50 \mathrm{~s}, m$ is reset to zero, below $m_{\text {low }}$ and the oscillators rest. No movement is generated.

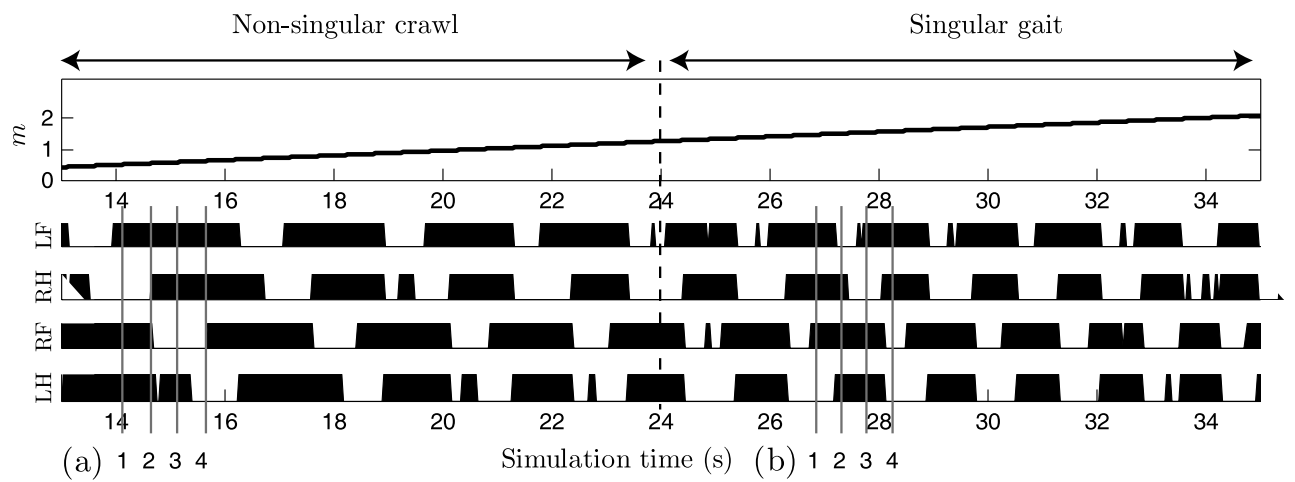

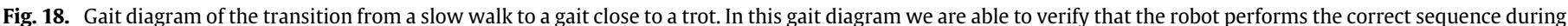
a walk (LF-RH-RF-LH). We are also able to visualize the gradual change on interlimb coordination and the decrease of duty factor.

during the continuous switch between the singular crawl to the trot gait.

Motor behaviors are either modulated or triggered by the $m$ modulatory drive signal provided to the second layer. In this experiment, this signal is modified online, at any time, according to sensory information. This signal is controlled by the capacitive touch sensors of the AIBO robot. Touching the back sensors in the back raises the modulatory drive. Touching the head sensor, decreases the modulatory drive. This means that switching between the possible locomotor behaviors is elicited by an external stimulus.

Fig. 20 illustrates the fast and smooth modulation of the trajectories according to parameter changes in real time. Fig. 21 presents snapshots depicting the experiment. A video from the experiment can be seen in the accompanying video.

Initially, the robot is performing a non-singular crawl, $\beta=$ 0.77, $\varphi_{\mathrm{LH}}=0.75$ (1-5 in Fig. 21). At $t=7 \mathrm{~s}$, the robot is stim- ulated during $1 \mathrm{~s}$ to increase its velocity, achieving the trot gait at $t=8 \mathrm{~s}, \beta=0.5$ and $\varphi_{\mathrm{LH}}=0.5$ (6-10 in Fig. 21). The fourth and bottom panels of Fig. 20 show the recorded servo joint encoders of the LF, LH and LF, RF, respectively. Trajectories are smoothly adjusted accordingly in less than a cycle, keeping the specified coordination between the limbs. Despite an increase in the frequency the limbs stay synchronized regarding its desired relative phases. Trajectories remain smooth despite a phase shift and a frequency adjustment.

A few seconds later, the head sensors are touched, stimulating the robot and reducing $m$ below 0.2 (Fig. 20, top panel). Accordingly, the $\mu$ parameter changes to a negative value (second panel), stopping the oscillators and the robot stops at $t=13.6 \mathrm{~s}$. This situation is depicted in snapshots 11 and 12 in Fig. 21.

Around $t=17.5 \mathrm{~s}$, the robot is again stimulated through a touch in the back sensors, raising $m$ to 1.62 (Fig. 20, top panel). $\beta$ (dark blue dotted line, third panel) and $\varphi_{\mathrm{LH}}$ (light yellow solid line, 

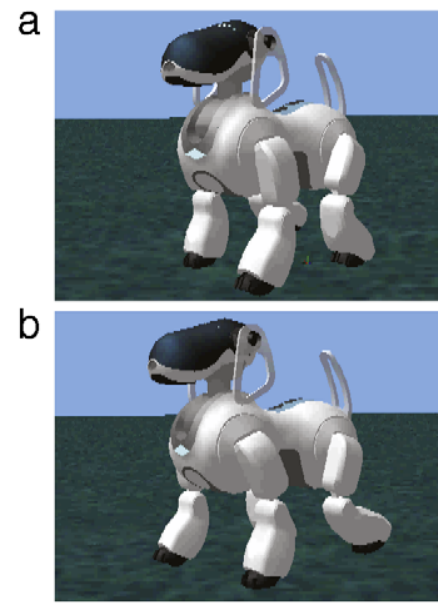

C

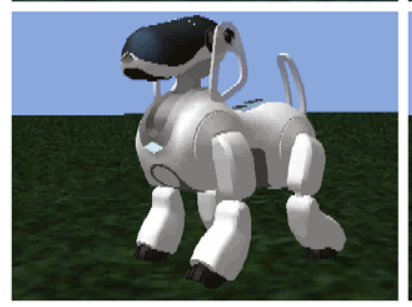

2
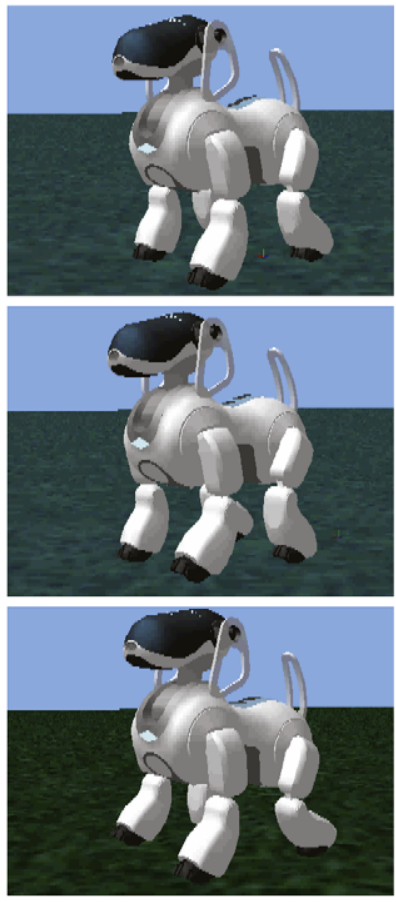
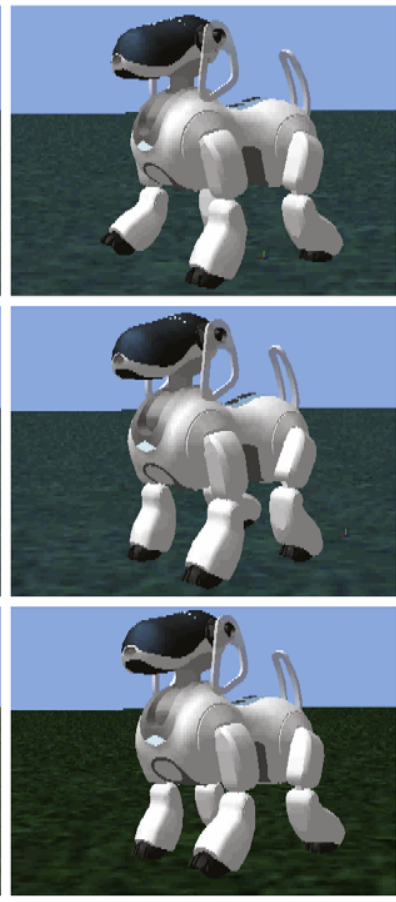

3
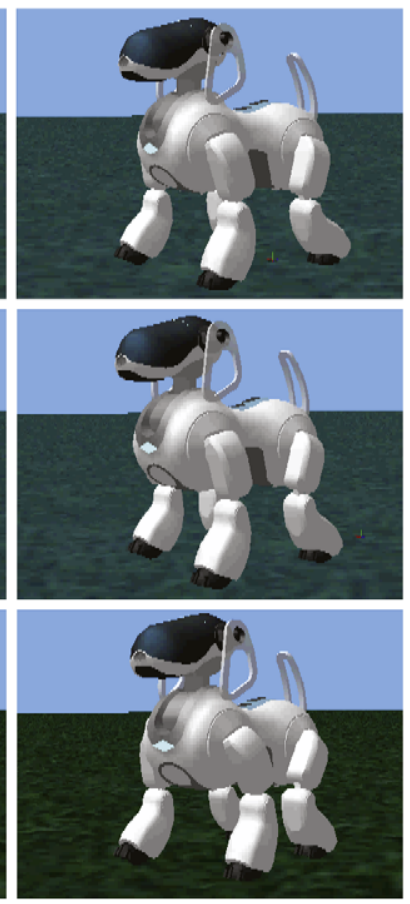

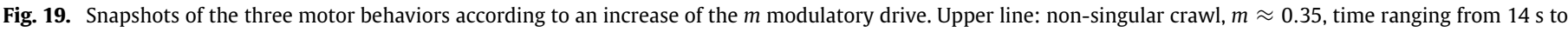

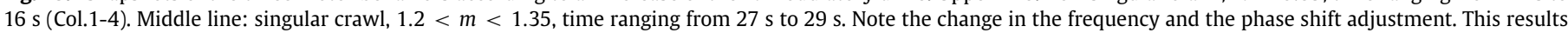

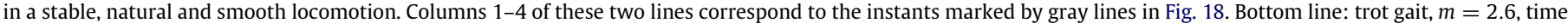
ranging from $44 \mathrm{~s}$ to $45 \mathrm{~s}$.

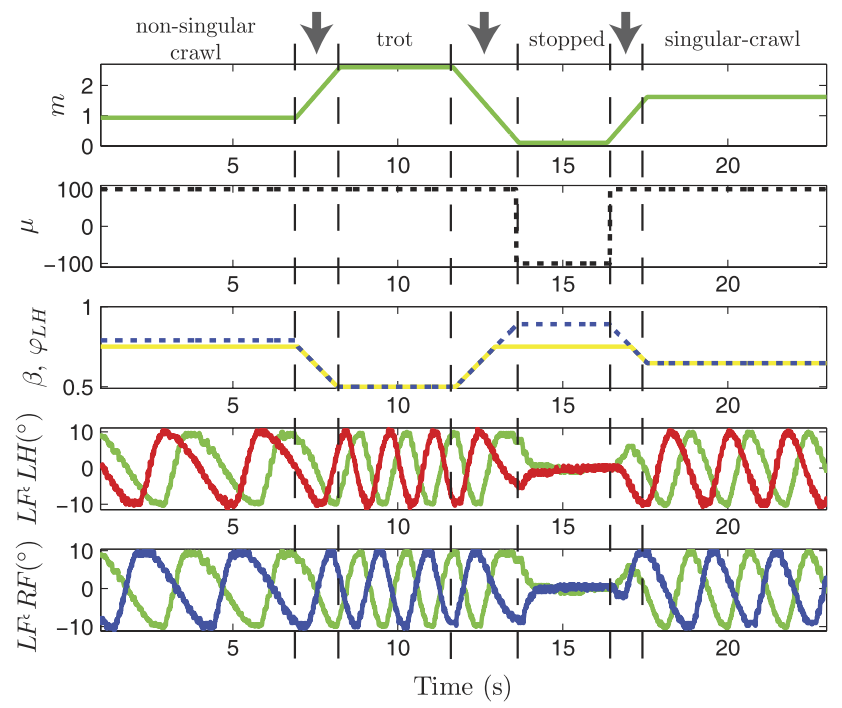

Fig. 20. Vertical arrows indicate time moments at which touch sensors, head or back, were stimulated. Accordingly, $m$ modulatory drive signal (top panel) and CPG parameters were changed. Second panel: $\mu$. Third panel: $\beta$ (dark blue dotted line) and $\varphi_{\mathrm{LH}}$ (light yellow solid line). Trajectories were recorded from the joints' encoders. Fourth panel: LH (red) and LF (light green) hip joint recorded trajectories. It shows the coordination of the left limbs changing according to the chosen gait phase $\varphi_{\mathrm{LH}}$. Bottom panel: RF (dark blue) and LF (light green) hip joint recorded trajectories. Note the coordination among these two contralateral limbs, always alternating.

third panel) change accordingly. The walking motion is resumed into a singular $\operatorname{crawl}\left(\beta=0.65, \varphi_{\mathrm{LH}}=0.65\right)$.

\section{Conclusions and future work}

In this work we have extended the use of nonlinear oscillators for locomotion generation in legged robotic platforms, by imple- menting a mechanism for controlling the velocity and gait selection using one simple command.

We propose a functionally stratified two-layer architecture. The second layer is responsible for controlling the lower rhythmic generation layer, i.e., able to initiate/stop and modulate the rhythmic activity. This idea is inspired by the vertebrate locomotor network [49], that receives excitatory commands and convergence from higher centers, and in turn sends the respective activation signals for the pattern generators. Specifically, the proposed layer controls the rhythmic generation of the locomotor movements, proportional to the strength of the received drive signal. By increasing the drive signal, locomotion can be elicited and velocity increased while switching to the appropriate gaits.

We further discuss and implement a gradual shift between gaits to improve the stability and the response of the robot during its locomotion, for the various velocities.

Three experiments are presented. In the first experiment, we compare two simulations in order to clarify the improvements when using a gradual transition between gaits. In the second experiment, we show how the robot system behaves when a drive signal set a priori initiates, stops and switches between different gaits. In the third experiment, locomotion is elicited through stimuli, mimicking the sensory pathways in nature. The system's modulatory drive signal is increased and decreased by touching the back and head of the robot, respectively. We also show the adequacy of the used dynamical systems in the generation of movement trajectories for the robot joints.

For generating adaptive locomotion we are currently addressing accurate feet placement; predictive adjustments of locomotion including speed and/or step length control in advance and head stabilization for image acquisition. This work should also be integrated with our previous work for posture and balance control [9].

Information from visual, sensory and vestibular sensors are also integrated in the reticulospinal region $[3,55]$, which then sends modulatory signals for the pattern generators. These ideas may be 

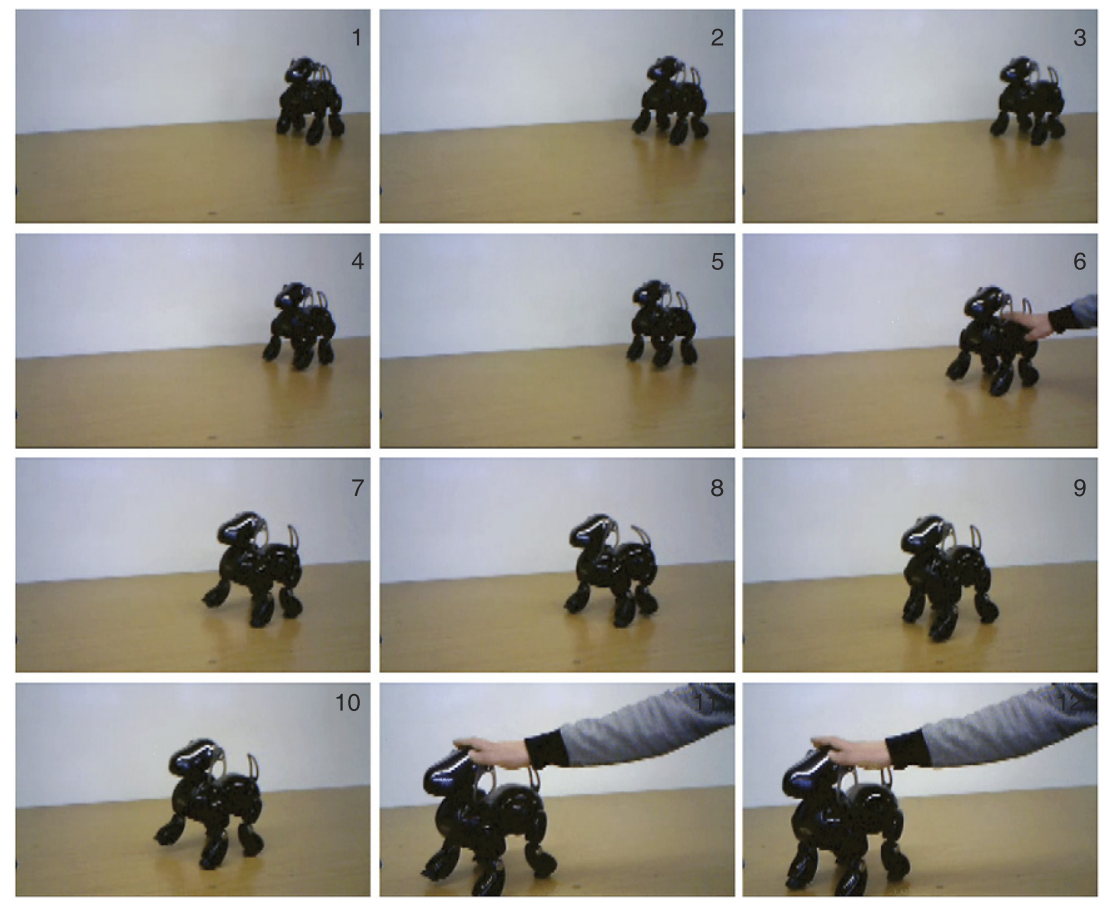

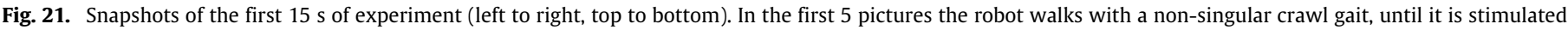

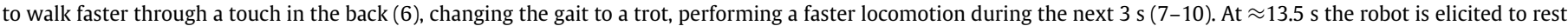
through a touch on the head (11), until it stops (12).

included in further development of our locomotor model, along with the development of mechanisms for motor program selection and fine-tuning movements, to name just a few.

\section{Acknowledgments}

The authors gratefully acknowledge Keir Pearson for all the discussions and help. This work is funded by FEDER Funding supported by the Operational Program Competitive Factors COMPETE and National Funding supported by the FCT - Foundation for Science and Technology through project PTDC/EEACRO/ $100655 / 2008$.

\section{References}

[1] S. Grillner, Locomotion in vertebrates: central mechanisms and reflex interaction, Physiological Reviews 55 (1975) 247-304.

[2] M. MacKay-Lyons, Central pattern generation of locomotion: a review of the evidence, Physical Therapy 82 (1) (2002) 69-83.

[3] S. Grillner, P. Wallna, K. Saitoha, A. Kozlova, B. Robertsona, Neural bases of goaldirected locomotion in vertebrates: an overview, Brain Research Reviews 57 (1) (2008) 2-12.

[4] S. Grillner, J. Hellgren, A. Ménard, K. Saitoh, M.A. Wikström, Mechanisms for selection of basic motor programs-roles for the striatum and pallidum, Trends Neuroscience 28 (7) (2005) 364-370. doi:10.1016/j.tins.2005.05.004.

[5] S. Degallier, L. Righetti, A. Ijspeert, Hand placement during quadruped locomotion in a humanoid robot: a dynamical system approach, in: IEEE-RAS International Conference on Intelligent Robots and Systems, IROS07, 2007.

[6] S. Degallier, L. Righetti, L. Natale, G.M.F. Nori, A. Ijspeert, A modular bioinspired architecture for movement generation for the infant-like robot icub, in: BioRob, 2008.

[7] L. Righetti, A.J. Ijspeert, Pattern generators with sensory feedback for the control of quadruped locomotion, in: 2008 IEEE International Conference on Robotics and Automation, 2008.

[8] S. Degallier, C. Santos, L. Righetti, A. Ijspeert, Movement generation using dynamical systems: a humanoid robot performing a drumming task, in: IEEERAS International Conference on Humanoid Robots, 2006.

[9] L. Castro, C. Santos, M. Oliveira, A. Ijspeert, Postural control on a quadruped robot using lateral tilt: a dynamical system approach, in: EUROS, in: Springer Tracts in Advanced Robotics, vol. 44, Springer, 2008, pp. 205-214.

[10] V. Matos, C. Santos, C. Pinto, A brainstem-like modulation approach for gait transition in a quadruped robot, in: 2009 IEEE IROS, 2009.
[11] R.B. Mcghee, A.A. Frank, On the stability properties of quadruped creeping gaits, Mathematical Biosciences 3 (1-2) (1968) 331-351.

[12] K. Inagaki, H. Kobayashi, A gait transition for quadruped walking machine, in: Proceeding of the 1993 IEEE/RSJ International Conference on Intelligent Robots and Systems.

[13] F. Hardarson, Stability analysis and synthesis of statically balanced walking for quadruped robots, Ph.D. Thesis, KTH (2002).

[14] S. Schaal, S. Kotosaka, D. Sternad, Nonlinear dynamical systems as movement primitives, in: First IEEE/RAS International Conference on Humaniod Robotics, Humanoids 2000, 2000.

[15] F. Delcomyn, Neural basis for rhythmic behaviour in animals, Science 210 (1980) 492-498.

[16] M.C. Trensch, P. Saltiel, E. Bizzi, The construction of movement by the spinal cord, Nature Neuroscience 2 (1999) 162-167.

[17] C. Liu, J. Su, Basic behavior acquisition based on multisensor integration of a robot head, in: ICRA, 2008, pp. 3094-3099.

[18] N. Kohl, P. Stone, Machine learning for fast quadrupedal locomotion, in: AAAI'04: Proceedings of the 19th National Conference on Artifical Intelligence, AAAI Press/The MIT Press, 2004, pp. 611-616.

[19] E. Bizzi, A. dAvella, P. Saltiel, M. Trensch, Modular organization of spinal motor systems, The Neuroscientist 8 (5) (2002) 437-442.

[20] G. Schöner, M. Dose, A dynamical systems approach to task-level system integration used to plan and control autonomous vehicle motion, Robotics and Autonomous Systems 10 (4) (1992) 253-267.

[21] A.J. Ijspeert, J. Nakanishi, S. Schaal, Learning attractor landscapes for learning motor primitives, in: Advances in Neural Information Processing Systems 15, MIT Press, 2002, pp. 1547-1554.

[22] G. Taga, Emergence of bipedal locomotion through entrainment among the neuro-musculo-skeletal system and the environment, in: Proceedings of the NATO Advanced Research Workshop and EGS Topical Workshop on Chaotic Advection, Tracer Dynamics and Turbulent Dispersion, Elsevier NorthHolland, Inc., New York, NY, USA, 1994, pp. 190-208. doi:10.1016/01672789(94)90283-6.

[23] R. Blickhan, The spring-mass model for running and hopping, Journal of Biomechanics 22 (11-12) (1989) 1217-1227.

[24] C. Gaskett, G. Cheng, Online learning of a motor map for humanoid robot reaching, in: Proceedings of the 2nd International Conference on Computational Intelligence, Robotics and Autonomous Systems, CIRAS 2003, 2003.

[25] C.P. Santos, Generating timed trajectories for an autonomous vehicle: a nonlinear dynamical systems approach., in: IEEE International Conference on Robotics and Automation, ICRA-2004, 2004.

[26] G. Schöner, C. Santos, Control of movement time and sequential action through attractor dynamics: a simulation study demonstrating object interception and coordination, in: SIRS 2001, 2001.

[27] C.P. Santos, M. Ferreira, Two vision-guided vehicles: temporal coordination using nonlinear dynamical systems, in: ICRA, 2007, pp. 14-19. 
[28] D.M. Wolpert, Z. Ghahramani, Perception, planning and control of autonomous walking with the ambler planetary rover, Nature Neuroscience 3 (2000) 1212-1217.

[29] H. Kimura, Y. Fukuoka, A.H. Cohen, Adaptive dynamic walking of a quadruped robot on natural ground based on biological concepts, International Journal of Robotics Research 26 (5) (2007) 475-490.

[30] M. Buhler, D.E. Koditschek, P.J. Kindlmann, Planning and control of a juggling robot, International Journal of Robotics Research 13 (2) (1994) 101-118.

[31] C. Santos, M. Oliveira, A.M.A. Rocha, L. Costa, Head motion stabilization during quadruped robot locomotion: combining dynamical systems and a genetic algorithm, in: IEEE International Conference on Robotics and Automation, 2009.

[32] C. Santos, M. Ferreira, Timed trajectory generation using dynamical systems: application to a puma arm, Robotics and Autonomous Systems 57 (2) (2009) 182-193. selected papers from 9th International Conference on Intelligent Autonomous Systems, IAS-9, 9th International Conference on Intelligent Autonomous Systems.

[33] K. Yoneda, S. Hirose, Dynamic and static fusion gait of a quadruped walking vehicle, Brain Research Reviews 9 (3) (1991) 7-15.

[34] A. Sano, J. Furusho, Static-dynamic transitional gait from crawl to pace, in: ROBOMEC'92, vol. B, 1992, pp. 239-246.

[35] H. Yasa, M. Ito, An autonomous decentralized system with application to a gait pattern generator, Transactions of the Society of Instrument and Control Engineers 26 (12) (1989) 180-187.

[36] E. Krotkov, R. Simmons, Perception, plaaning and control of autonomous walking with the ambler planetary rover, IEEE Transactions on Robotics and Automation 15 (1996) 155-180.

[37] A.J.I. Aude Billard, Biologically inspired neural controllers for motor control in a quadruped robot, in: Proceedings of the International Joint Conference on Neural Networks, vol. 6, 2000, p. 6637.

[38] A.O.K. Tsujita, K. Tsuchiya, Adaptive gait pattern control of a quadruped locomotion robot, in: Proc. IEEE/RSJ Int. Conf. on Intell. Robots Syst., vol. 6, 2001, pp. 2318-2325.

[39] J.-N.L.S.-M. Song, Modeling gait transitions of quadruped based on gait kinematics and cmac neural networks, in: Proceedings IEEE World Congress on Computational Intelligence, 1998, pp. 2075-2080.

[40] M. Raibert, K. Blankespoor, G. Nelson, Rob Playter, The Big-Dog Team, Bigdog, the rough-terrain quadruped robot, in: Proceedings of the 17th World Congress The International Federation of Automatic Control, 2008.

[41] P.B.B.J.M.P.J. Rebula, J.R. Neuhaus, A controller for the little dog quadruped walking on rough terrain, in: ICRA 2007, 2007.

[42] D.R.A.J. Ijspeert, A. Crespi, J.-M. Cabelguen, From swimming to walking with a salamander robot driven by a spinal cord model, Science 315 (5817) (2007) 1416-1420.

[43] J. Buchli, A.J. Ijspeert, Distributed central pattern generator model for robotics application based on phase sensitivity analysis, in: Biologically Inspired Approaches to Advanced Information Technology: First International Workshop, BioADIT 2004, Lectures Notes in Computer Science, 2004.

[44] K. Tsujita, H. Toui, K. Tsuchiya, Dynamic turning control of a quadruped locomotion robot using oscillators, Advanced Robotics 19 (October) (2005) 1115-1133 (19).

[45] G.K. Pearson, Generating the walking gait: role of sensory feedback, Progress in Brain Research 143 (2004) 123-129.
[46] O. Kiehn, Locomotor circuits in the mammalian spinal cord, Annual Review of Neuroscience 29 (2006) 279-306. doi:10.1146/annurev.neuro.29.051605.112910.

[47] S. Grillner, Control of locomotion in bipeds, tetrapods and fish, in: V. Brooks (Ed.), Handbook of Physiology, The Nervous System, 2, Motor Control American Physiology Society, Bethesda, 1981, pp. 1179-1236.

[48] S. Rossignol, R. Dubuc, J.P. Gossard, Dynamic sensorimotor interactions in locomotion, Physiological Review 86 (1) (2006) 89-154.

[49] M. Shimamura, S. Grillner, V.R. Edgerton, L.M. Jordan, Brainstem and spinal cord mechanisms for the initiation of locomotion, in: Neurobiological Basis of Human Locomotion Tokyo Japan Scientific Societies, 1991.

[50] A. Ijspeert, A. Crespi, D. Ryczko, J.-M. Cabelguen, From swimming to walking with a salamander robot driven by a spinal cord model, Science 315 (5817) (2007) 1416-1420.

[51] C.D. Marsden, P. Merton, H. Morton, The use of peripheral feedback in the control of movements, Trends Neuroscience (7) (1984) 253-258.

[52] O. Michel, Webots: professional mobile robot simulation, International Journal of Advanced Robotic Systems 1 (1) (2004) 39-42.

[53] S.-M. Song, K.J. Waldron, Machines That Walk: The Adaptive Suspension Vehicle, MIT Press, Cambridge, MA, USA, 1988.

[54] C.P. Santos, V. Matos, http://dei-s1.dei.uminho.pt/asbgroup/uploads/journalsimulation, 2009.

[55] T. Deliagina, I. Beloozerova, P. Zelemin, G. Orlovsky, Spinal and supraspinal postural networks, Brain Research Reviews 57 (2008) 212-221.

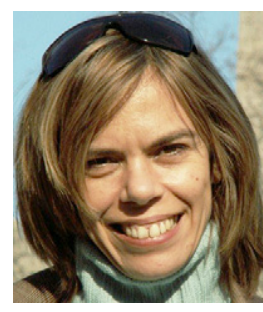

Cristina P. Santos Doctor Santos received the B.S. degree in Industrial Electronics, the M.Sc degree in Robotics, and the Ph.D. degree in Robotics in the field of nonlinear dynamics, all from the University of Minho, Guimaraes, Portugal, in 1994, 1998 and 2003 respectively. The Ph.D. was also in collaboration with the CNRS-CNRC Marseille, France.

She is currently working as an Auxiliar Professor at the University of Minho, Industrial Electronics Department. Her research focus on the extension of the use of dynamical systems theory to the achievement of more complex behavior for robots: generate locomotion for multi-DOF robots; and achieve cooperativity among multi-robots and learning.

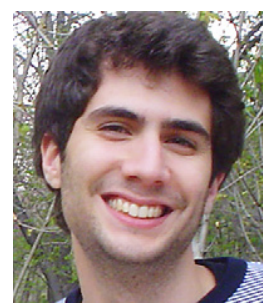

Vitor Matos is a Ph.D. student in Robotics at the University of Minho. He holds a M.S. degree in Industrial Electronics from the University of Minho since 2009. Current research includes robot locomotion and dynamical system theory for achieving generation of motions and behaviors. 